\title{
Efecto de la densidad de siembra en la productividad y rentabilidad del langostino Macrobrachium rosenbergii en la fase de engorde en estanques, Costa Rica
}

\author{
Effect of stocking density on the productivity and profitability of the freshwater \\ prawn Macrobrachium rosenbergii in the fattening phase in ponds, Costa Rica
}

\author{
José Valverde ${ }^{1,3}$, Alexander Varela ${ }^{2,4}$
}

\section{Resumen}

El objetivo del presente estudio fue determinar la productividad y rentabilidad del engorde de langostinos cultivados en dos densidades de siembra en estanques, aplicando una tecnología sencilla y apropiada para la transferencia de conocimientos a los productores. El estudio se llevó a cabo en 2019 en una finca acuícola en la provincia de Limón, Costa Rica. La etapa de engorde se realizó usando densidades de 2.5 y 6.0 langostinos $/ \mathrm{m}^{2}$. Las producciones obtenidas fueron de 758.9 y $961.2 \mathrm{~kg} / \mathrm{ha}$, respectivamente $(\mathrm{p}<0.01)$. Asumiendo 2.7 ciclos al año, la productividad potencial sería de 2049 y $2595 \mathrm{~kg} / \mathrm{ha} /$ año de manera respectiva. Los costos variables de producción fueron más altos a $6.0 / \mathrm{m}^{2}$ que a $2.5 / \mathrm{m}^{2}$ por el incremento en la compra de mayor cantidad de semilla y de alimento. El costo de la semilla representó el $69 \%$ de la inversión total en baja densidad de siembra y $77 \%$ en la más alta. Los ingresos por ventas en granja fueron más altos a $6.0 / \mathrm{m}^{2}$ que a $2.5 / \mathrm{m}^{2}$. No obstante, la densidad de siembra más rentable fue la de $2.5 / \mathrm{m}^{2}$ debido a que aún con una menor producción, dio una utilidad mayor. Las utilidades brutas fueron mayores en la densidad de siembra más baja, y no hubo rentabilidad en la más alta, debido no solo a la mayor proporción de langostinos con talla comercial en la primera densidad, sino también al aumento en el costo de producción en la segunda, donde la semilla representó el mayor gasto. El estudio de sensibilidad económica sugiere la utilización de la densidad de siembra de $2.5 / \mathrm{m}^{2}$ por tener mayor rentabilidad, una TIR

\footnotetext{
${ }^{1}$ Instituto Nacional de Aprendizaje, Núcleo Náutico Pesquero, Puntarenas. Costa Rica

${ }^{2}$ Consultor en Diagnóstico y Sanidad Acuícola, Sonora, México

${ }^{3}$ E-mail: jvalverdemoya@ina.ac.cr

${ }^{4}$ E-mail: alexander.varela@gmail.com
}

Recibido: 22 de octubre de 2019

Aceptado para publicación: 10 de junio de 2020

Publicado: 11 de agosto de 2020 
(48.6\%) atractiva, relativamente poco tiempo para recuperar la inversión (2.1 años), un VAN positivo y una relación beneficio costo de 2.0 lo que significa que por cada dólar invertido se generaron US\$2.0.

Palabras clave: langostinos, Macrobrachium rosenbergii, densidad de siembra, productividad, rentabilidad

\section{AbSTRACT}

The aim of this study was to determine the productivity and profitability of fattening freshwater prawns cultivated in two stocking densities in ponds, applying a simple and appropriate technology for the transfer of knowledge to producers. The study was carried out in 2019 on a farm in the province of Limón, Costa Rica. The fattening stage was carried out using densities of 2.5 and $6.0 \mathrm{shrimp} / \mathrm{m}^{2}$. The productions obtained were 758.9 and $961.2 \mathrm{~kg} / \mathrm{ha}$, respectively $(\mathrm{p}<0.01)$. Assuming 2.7 cycles per year, the potential productivity would be 2049 and $2595 \mathrm{~kg} / \mathrm{ha} /$ year, respectively. Variable production costs were higher at $6.0 / \mathrm{m}^{2}$ than at $2.5 / \mathrm{m}^{2}$ due to the increase in the purchase of more seeds and feeds. The cost of the seed represented $69 \%$ of the total investment in low stocking density and $77 \%$ in the highest. Farm sales revenue was higher at $6.0 / \mathrm{m}^{2}$ than at $2.5 / \mathrm{m}^{2}$. However, the most profitable stocking density was $2.5 / \mathrm{m}^{2}$ because even with lower production, it showed a higher profit. Gross profits were higher in the lowest stocking density, and there was no profitability in the highest, due not only to the higher proportion of prawns with commercial size in the first stock, but also to the increase in the cost of production in the second, where the seed represented the greatest expense. The economic sensitivity study suggests the use of the seeding density of $2.5 / \mathrm{m}^{2}$ has higher profitability, an attractive IRR (48.6\%), relatively less time to recover the investment (2.1 years), a positive NPV and a cost benefit ratio. 2.0 which means that for every dollar invested US $\$ 2.0$ was generated.

Key words: prawns, Macrobrachium rosenbergii, planting density, productivity, profitability

\section{INTRODUCCIÓN}

El 98\% de la producción mundial del langostino de agua dulce Macrobrachium rosenbergii se obtenía en el año 2000 de los países asiáticos y el restante $2 \%$ se desarrollaba en algunos países del continente americano, especialmente de Brasil, México, Costa Rica y EEUU (New, 2000; New y Nair, 2012). Estos países recibieron pequeñas cantidades de reproductores, lo que les hizo más vulnerables, posiblemente debido a una disminución en la variabilidad genética (New y Kutty, 2010).

La producción de langostinos en Costa Rica se inició en 1979 con reproductores introducidos desde Honduras por parte de la Misión de la República de Taiwán (Valverde, 1987) y desde Hawaii y Florida (New, 2010) por la empresa Langostinos KoKo. Esta empresa obtuvo producciones de 84 toneladas métricas (t) anuales en 1996 y 1997, pero bajó 
drásticamente en el tiempo, manteniéndose con producciones bastante reducidas (New y Kutty, 2010). Actualmente Langostinos KoKo no produce a nivel comercial y se dedica únicamente a la reproducción y venta de pos-larvas (Pl's).

Desde 2002, Costa Rica solo produce langostinos en pequeña escala para el consumo local o familiar y hasta el presente no ha existido el desarrollo exponencial esperado para esta especie, aunque se mantiene el interés y las condiciones ambientales óptimas para su cultivo (Peña y Chacón, 2019). Actualmente, dos criaderos son responsables de suministrar semilla a todos los productores nacionales. Las etapas de criadero se han mejorado recientemente después de superar algunas limitantes que estaban restringiendo la eficiencia en la reproducción y producción de Pl's (Varela-Mejías y Valverde-Moya, 2018).

El cultivo de langostinos presenta diversos problemas que vienen afectando su producción en varios países, incluido Tailandia (Na Nakorn y Jintasatapom, 2012) o la han mantenido estática (New, 2000), como aquellos relacionados a la sobrevivencia y crecimiento de juveniles y adultos en estanques de producción. Además, los periodos de cultivo son muy prolongados (8-12 meses), hay saturación de mercados, altos costos de producción (semilla y alimento) y variable calidad de las Pl's. También existe competencia en el mercado con otras especies como tilapias (Oreochromis sp) y camarones marinos (Penaeus vannamei) cultivados en agua dulce (Na-Nakorn, 2017).

Dado que la producción y el crecimiento de los langostinos dependen de la densidad de siembra (Karplus et al., 2000), es prioritario evaluar el efecto de diferentes densidades en la productividad y rentabilidad, considerando que el precio depende del tamaño del producto. Por ello, este estudio evaluó la productividad y rentabilidad a diferentes densidades de siembra del langostino de agua dulce $M$. rosenbergii como parte del paquete tecnológico que se necesita para el cultivo de langostinos en Costa Rica.

\section{Materiales y Métodos}

El estudio se llevó a cabo desde enero hasta mayo de 2019 en una finca acuícola ubicada en el distrito La Rita $\left(10^{\circ} 12^{\prime} 56^{\prime \prime} \mathrm{N}\right.$ y $83^{\circ} 47^{\prime} 32^{\prime \prime}$ O) del cantón de Pococí, en la región Caribe de la provincia de Limón, Costa Rica. El estudio económico comparó dos densidades de siembra: 2.5 langostinos $/ \mathrm{m}^{2}$ y 6.0 langostinos $/ \mathrm{m}^{2}$ en dos estanques de $300 \mathrm{~m}^{2}$ $(10 \times 30 \mathrm{~m})$, cada uno dividido a la mitad por una red de malla mosquitero enterrada en el fondo. Cada sección tuvo un área de $150 \mathrm{~m}^{2}$ y las secciones se numeraron del 1 al 4 para los efectos del estudio. La designación de cada sección con su respectiva densidad se hizo intercambiando entre ellas la posición de la parte superior menos profunda y la inferior cerca de la salida del agua con mayor columna de agua, esto con el fin de evitar un posible sesgo por esta condición. Por lo tanto, se sembraron 900 juveniles en la densidad de siembra de $6.0 / \mathrm{m}^{2}$ y 375 en la densidad de $2.5 / \mathrm{m}^{2}$. Extrapolando, se trata de densidades de siembra de 60000 y 25000 langostinos por hectárea, respectivamente.

Los estanques estaban recién construidos y se utilizó agua proveniente del nivel freático del fondo de estos. Por tal motivo, no se realizó ningún tipo de preparación; práctica común en estanques abastecidos con agua superficial como el secado, desinfección y filtración del agua en el llenado. Tampoco se fertilizaron sus aguas debido a que adquirieron la turbidez y coloración verde característica de la proliferación del fitoplancton desde antes de la siembra. Los niveles del agua no fueron controlados debido a que dependían del nivel de aguas subterráneas, así como de la cantidad de lluvias y horas sol. En general, se mantuvo $0.5 \mathrm{~m}$ en la parte más baja y $0.8 \mathrm{~m}$ en la más profunda con un promedio de $0.7 \mathrm{~m}$. 
Los estanques se sembraron con juveniles de 3 meses de edad, provenientes de un tanque de $10 \mathrm{~m}^{3}$ usado como pre-cría en una instalación cercana $(100 \mathrm{~m})$. Los juveniles se cosecharon con un peso de $1.0 \pm 0.8 \mathrm{~g}$ (rango: $0.1-4.1 \mathrm{~g}$ ). A simple vista fue posible distinguir dos poblaciones definidas (grandes y pequeños). En este estudio se utilizaron los juveniles grandes con peso de $1.7 \pm 0.8 \mathrm{~g}$, con un mínimo de $1.0 \mathrm{~g}$ y un máximo de $4.1 \mathrm{~g}$.

Posterior a la siembra de los langostinos, semanalmente se midió el oxígeno disuelto y la temperatura del agua dos veces por día (06:00 y 16:00) a $10 \mathrm{~cm}$ del fondo utilizando el dispositivo Hanna HI9146. Se midió la turbidez del agua a las 10:00 horas con un disco Secchi. El pH, la alcalinidad, la dureza y las concentraciones de amonio, nitritos, nitratos y fósforo se determinaron mensualmente siguiendo el procedimiento estandarizado para esas determinaciones (Lazur, 2007).

El muestreo de los langostinos se hizo quincenalmente extrayendo una muestra no selectiva de cada estanque utilizando una atarraya. Los langostinos se depositaron en baldes plásticos, se mantuvieron con aireación constante, se sexaron, se pesaron en una balanza electrónica Ohaus $( \pm 0.05 \mathrm{~g})$ y se midieron (desde el borde anterior del ojo hasta la punta del telson) con una regla graduada en $\mathrm{mm}( \pm 0.5 \mathrm{~mm})$. Después se regresaron a sus respectivos estanques.

Los langostinos fueron alimentados diariamente con balanceado en pellets para camarones marinos (Biomar), producido a nivel nacional y con $30 \%$ de proteína. La dosis diaria y la frecuencia de la alimentación se calcularon tomando en cuenta el peso promedio y la biomasa estimada. El alimento se suministró en tres raciones al día hasta el primer mes de cultivo y luego en dos raciones al día.

La cosecha se hizo a los 4 meses de la siembra ( 7 meses de edad). Se realizó pasando una red y drenando el agua en los estanques con una motobomba para colectar los remanentes con la mano o con atarraya. Se depositaron en baldes con aireación para contarlos, medirlos, pesarlos y clasificarlos por sexo y morfotipo. Los machos se clasificaron en pequeños con menos de $10 \mathrm{~g}$, medianos de tenazas anaranjadas de 10-25 g y grandes de tenazas azules con más de $25 \mathrm{~g}$, mientras que las hembras se clasificaron según su estado de gravidez (pre-ovígeras, ovígeras y pos-ovígeras).

La productividad se evaluó comparando los promedios del peso (g), la conversión alimenticia (kg alimento/kg langostinos), la producción final $(\mathrm{kg})$ y la biomasa total relativa (biomasa final/biomasa inicial) al final del estudio. Los datos fueron analizados con la prueba de T de Student (Bhujel, 2008) por medio del paquete estadístico SAS. Se planteó la hipótesis negativa de que no había diferencia significativa entre los tratamientos de las diferentes densidades de siembra con una confiabilidad del $99 \%$. La producción final se caracterizó considerando el tamaño comercial ( $>30 \mathrm{~g})$ y el no comercial $(<30 \mathrm{~g})$ obteniéndose la proporción de langostinos en cada clase por sexo y morfotipo. La diferencia significativa entre estas proporciones se obtuvo aplicando la distribución de Chi cuadrado. La productividad se expresó en $\mathrm{kg} / \mathrm{ha} / \mathrm{año}$ en cada densidad de siembra.

La rentabilidad se calculó para el cultivo de langostinos en estanques de tierra en las condiciones ambientales, de calidad del agua y de manejo establecidas en esta investigación. El registro de los costos de producción se trasladó a un tipo de actividad productiva a nivel comercial con base en la experiencia adquirida en la empresa Langostinos KoKo (New y Kutty, 2010).

El costo unitario de producción se obtuvo tomando en cuenta los costos fijos de infraestructura y depreciación para un estanque de 1 ha durante una vida económica de 10 años del proyecto a nivel comercial. También se consideraron los costos de operación y manejo pos-cosecha de los langostinos para la venta. Los ingresos se establecieron se- 
gún el precio del producto entero $(\mathrm{g})$ y fresco al pie de finca de acuerdo con las características en que fueron clasificados. Las ganancias se calcularon considerando la biomasa producida $(\mathrm{kg})$ y el precio según el tamaño del producto. El precio por kilo se obtuvo de una comunicación personal por parte de Harold Phillips y Álvaro Montero, propietarios de viveros y fincas de producción en Sardinal de Carrillo, Guanacaste y Barrio Jesús María de San Mateo, Alajuela, respectivamente.

La rentabilidad del engorde de langostinos se obtuvo según el punto de equilibrio, el cual indica el valor del producto (costo variable de producción/producción) y el nivel de producción (costo variable de producción/precio por kilo) en el que los ingresos apenas alcanzan para cubrir los costos. También se obtuvo la utilidad por hectárea por año, la TIR (Tasa Interna de Retorno), el VAN (Valor Actual Neto) y la relación beneficio/costo actualizados a un factor de descuento del $28 \%$ para la densidad de siembra que dio rentabilidad.

\section{Resultados}

\section{Parámetros Ambientales y de la Calidad del Agua}

Los valores promedios mensuales \pm desviación estándar de la humedad relativa, temperatura ambiental, precipitación y evaporación, medidos en la Estación Meteorológica de CORBANA en La Rita de Pococí, Limón, ubicada a $3 \mathrm{~km}$ del sitio de estudio, fueron de $82.6 \pm 2.4 \%, 24.7 \pm 0.8^{\circ} \mathrm{C}, 171.1$ $\pm 16.4 \mathrm{~mm}$ y $123.6 \pm 22.9 \mathrm{~mm}$, respectivamente, durante el periodo del estudio. La temperatura y el oxígeno disuelto del agua en los dos estanques presentaron valores de $25.0 \pm$ $1.6{ }^{\circ} \mathrm{C}$ y $27.8 \pm 2.1{ }^{\circ} \mathrm{C}$ y de $4.9 \pm 1.3 \mathrm{mg} / \mathrm{l}$ y $7.6 \pm 1.8 \mathrm{mg} / 1$, respectivamente. Antes de la siembra, estos valores eran de $25.3{ }^{\circ} \mathrm{C}$ y 4.5 $\mathrm{mg} / 1$ en las mañanas. La turbidez promedio fue de $48 \pm 14 \mathrm{~cm}$ y el $\mathrm{pH}$ del agua fue de 7.7 \pm 1 .2. Otros parámetros químicos medidos en los dos estanques fueron: nitratos $(0.03$ $\mathrm{mg} / \mathrm{l})$ nitritos $(0.03 \mathrm{mg} / \mathrm{l})$, nitrógeno amoniacal $(0.53 \mathrm{mg} / \mathrm{l})$, amoníaco $(0.02 \mathrm{mg} / \mathrm{l})$, fosfatos $(0.2 \mathrm{mg} / \mathrm{l})$, alcalinidad $\left(61.4 \mathrm{mg} / \mathrm{l} \mathrm{CaCO}_{3}\right)$, dureza total $\left(72.6 \mathrm{mg} / 1 \mathrm{Ca} \mathrm{CO}_{3}\right)$ y por calcio $(43.6 \mathrm{mg} / \mathrm{l})$.

\section{Rendimientos de Producción}

En la Figura 1 se presentan los pesos (g) y las producciones $\left(\mathrm{g} / \mathrm{m}^{2}\right)$ promedios finales de los langostinos para las dos densidades de siembra. El peso promedio final fue significativamente superior $(\mathrm{p}<0.01)$ para los langostinos en la densidad de siembra de 2.5 organismos $/ \mathrm{m}^{2}(34.0 \mathrm{~g})$ en comparación con la de 6.0 organismos $/ \mathrm{m}^{2}(20.1 \mathrm{~g})$, producto del mayor crecimiento diario en cada caso ( $0.27 \mathrm{vs} 0.16 \mathrm{~g} / \mathrm{d}$, respectivamente). La producción promedio fue mayor en los langostinos sembrados en la densidad de a 6.0 organismos $/ \mathrm{m}^{2}\left(96.1 \mathrm{~g} / \mathrm{m}^{2}\right)$ que en aquellos sembrados a 2.5 organismos $/ \mathrm{m}^{2}\left(75.9 \mathrm{~g} / \mathrm{m}^{2}\right)$ $(\mathrm{p}<0.01)$.

La tasa de sobrevivencia fue significativamente superior $(p<0.01)$ en la densidad baja de siembra (89\%) que en la alta densidad de siembra $(80.1 \%)$ El descenso de la sobrevivencia en cada densidad de siembra se presenta en la Figura 2. La sobrevivencia fue calculada considerando la tasa instantánea de mortalidad y el conteo de los langostinos al final del cultivo. Al no detectarse organismos muertos en el transcurso del experimento, se presume que estos fueron depredados por ellos mismos. La conversión alimenticia fue de $1.6: 1.0$ a $2.5 / \mathrm{m}^{2}$ y $2.2: 1.0$ a $6.0 / \mathrm{m}^{2}$, sin existir diferencias significativas entre grupos, mientras que las tasas de alimentación fueron de 15.1 y $25.9 \mathrm{~g} / \mathrm{m}^{2} / \mathrm{d}$, respectivamente.

La producción final se da en términos de la biomasa en el estanque como resultado de un balance entre el aumento en peso y la mortalidad de los organismos sembrados. Con la sobrevivencia estimada en función del tiem- 


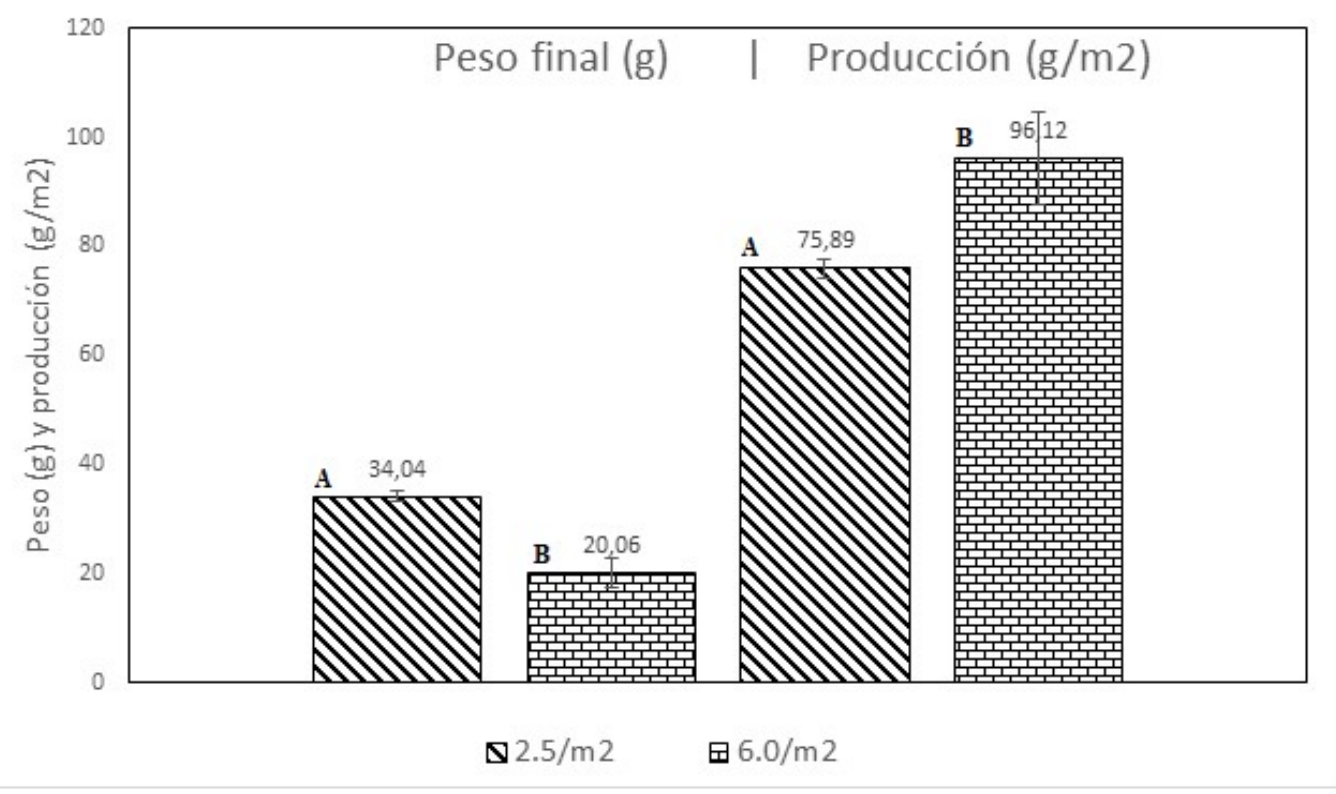

Figura 1. Peso (g) y biomasa $\left(\mathrm{g} / \mathrm{m}^{2}\right)$ final de langostino de agua dulce Macrobrachium rosenbergii en dos densidades de siembra. Variables con letras diferentes (A, B) indican diferencia significativa $(\mathrm{p}<0.01)$

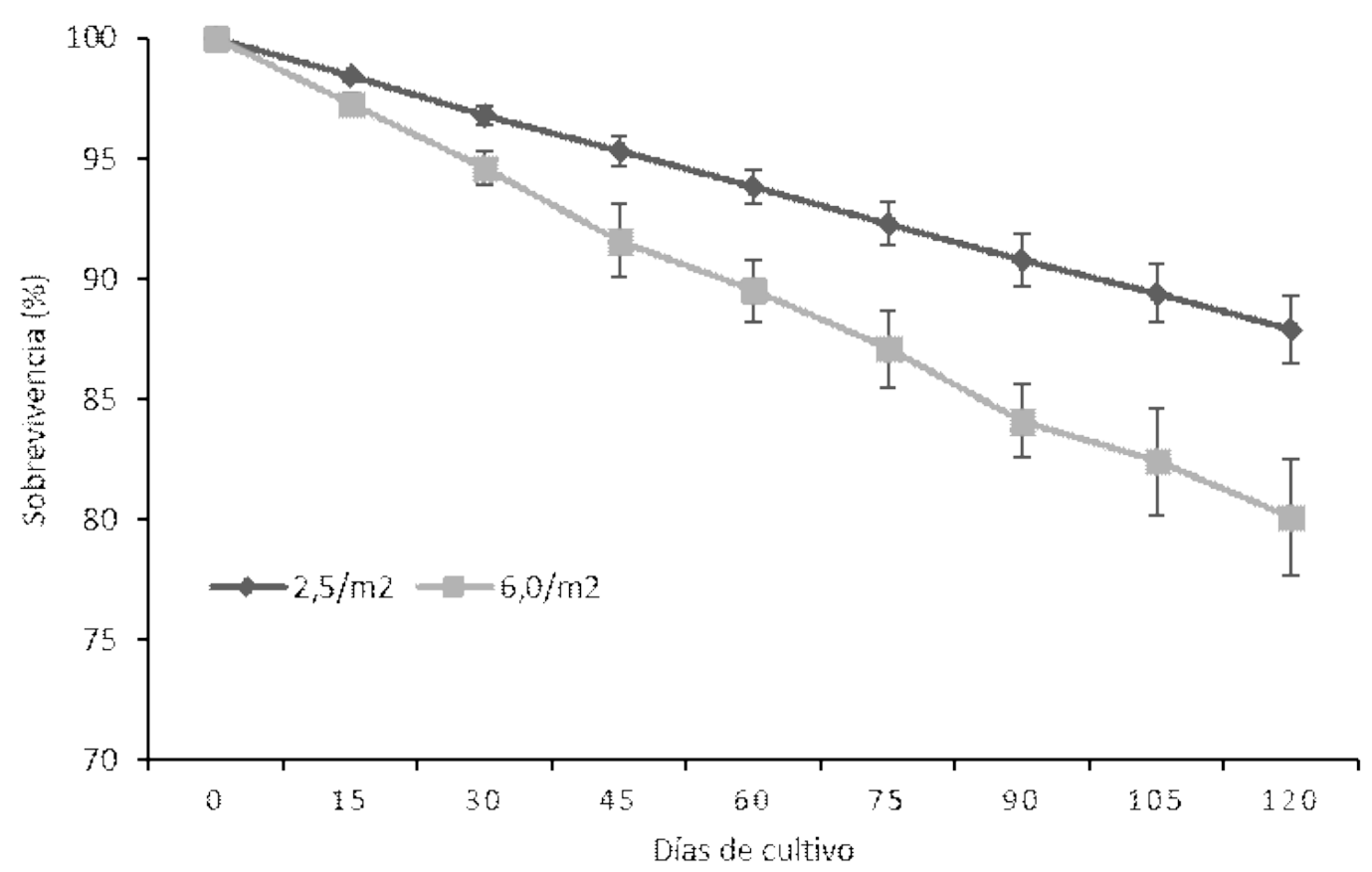

Figura 2. Sobrevivencia estimada en función del tiempo de langostinos de agua dulce Macrobrachium rosenbergii bajo dos densidades de cultivo 


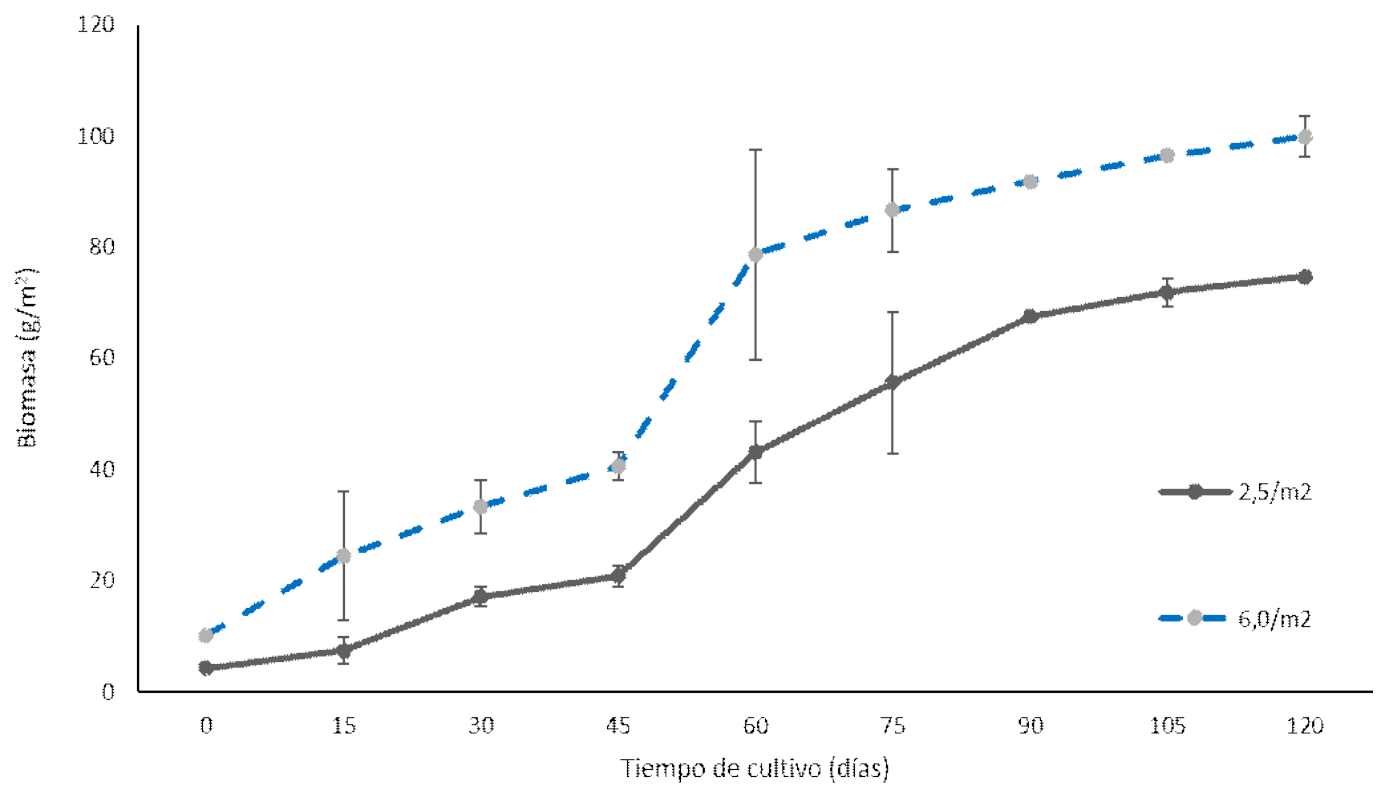

Figura 3. Incremento en la biomasa $\left(\mathrm{g} / \mathrm{m}^{2}\right)$ de langostinos de agua dulce Macrobrachium rosenbergii bajo dos densidades de cultivo

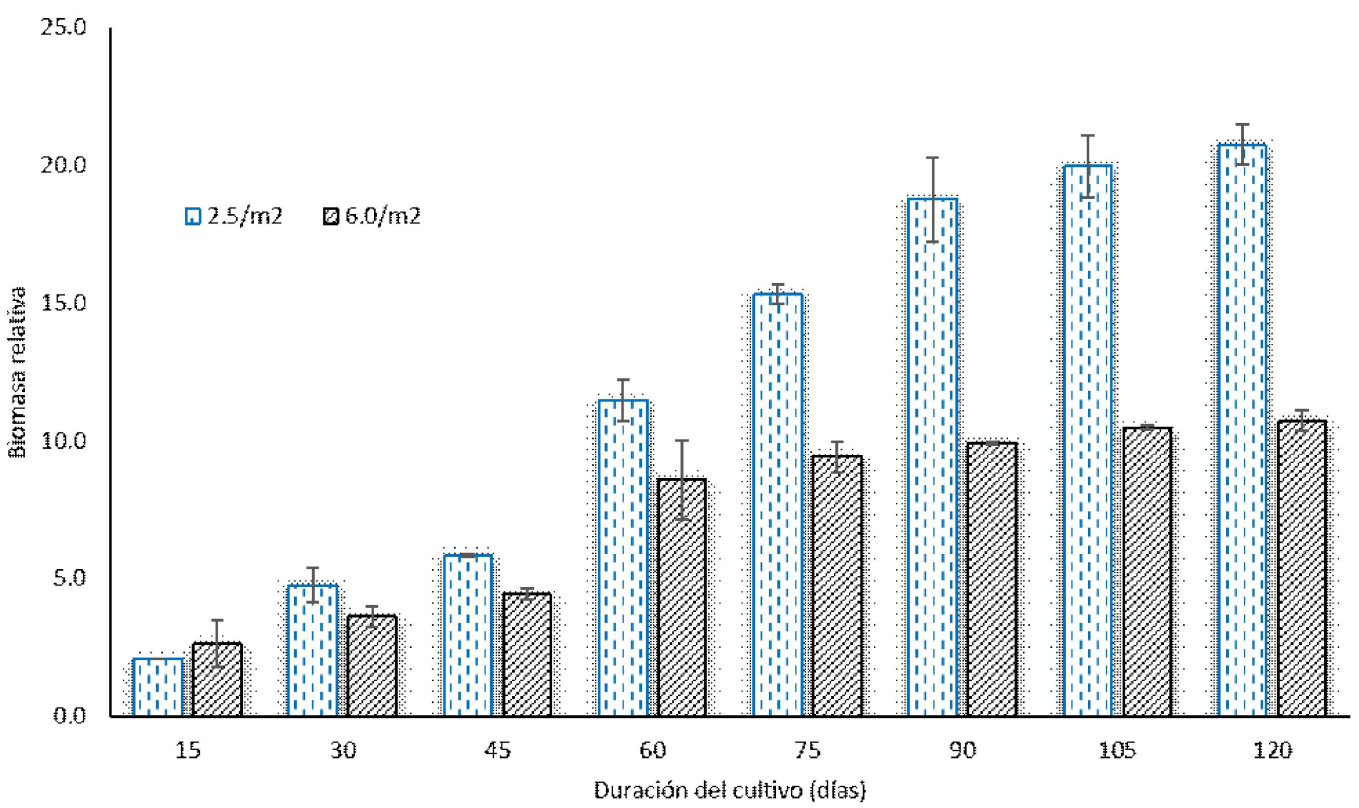

Figura 4. Biomasa relativa en función del tiempo para langostinos de agua dulce Macrobrachium rosenbergii bajo dos densidades de cultivo 
po y los muestreos quincenales del peso se pudo establecer la curva de biomasa (Figura 3). Se aprecia la mayor producción de los langostinos sembrados a $6 / \mathrm{m}^{2}\left(96.1 \mathrm{~g} / \mathrm{m}^{2}\right)$ con relación a los sembrados a $2.5 / \mathrm{m}^{2}(75.9$ $\left.\mathrm{g} / \mathrm{m}^{2}\right)(\mathrm{p}<0.01)$, diferencia atribuible a la mayor cantidad de langostinos sembrados en el primer caso, lo cual aumenta la producción, pero reduce el tamaño de los langostinos a la cosecha.

Para obviar este efecto, se calculó la biomasa total relativa dividiendo la biomasa acumulada en determinado periodo de tiempo entre la biomasa inicial. Esta indica el número de veces que se incrementó la biomasa inicial a través del tiempo. En la Figura 4 se puede observar que los valores promedios finales de la biomasa total relativa fueron prácticamente el doble para los langostinos sembrados a $2.5 / \mathrm{m}^{2}$ con respecto a los sembrados a $6.0 / \mathrm{m}^{2}$ (21 y 11 veces la biomasa inicial, respectivamente) $(\mathrm{p}<0.01)$.

En términos de producción comercial, los datos obtenidos por unidad de área $\left(\mathrm{m}^{2}\right)$ se deben extrapolar a la unidad de hectárea por ser la comúnmente utilizada. Por lo tanto, las producciones extrapoladas fueron de 758.9 y $961.2 \mathrm{~kg} / \mathrm{ha}$ a $2.5 / \mathrm{m}^{2}$ y $6.0 / \mathrm{m}^{2}$, respectivamente $(\mathrm{p}<0.01)$. No obstante, el rendimiento de producción en términos de kilos producidos por cada millar de juveniles sembrados fue casi el doble en la primera densidad (30.4 $\mathrm{kg} / \mathrm{millar}$ ) que en la segunda ( $16.0 \mathrm{~kg} / \mathrm{millar})$. Con una duración de 120 días en el engorde de langostinos, como en este caso de estudio, y asumiendo 15 días entre preparación de estanques, se pueden hacer 2.7 ciclos al año. Por lo tanto, la productividad sería de $2049 \mathrm{~kg} / \mathrm{ha} / \mathrm{anno}$ a $2.5 / \mathrm{m}^{2}$ y $2595 \mathrm{~kg} / \mathrm{ha} /$ año a $6.0 / \mathrm{m}^{2}$.

\section{Crecimiento y Dimorfismo Sexual}

El crecimiento de los langostinos se muestra en la Figura 5. Los langostinos sembrados a $2.5 / \mathrm{m}^{2}$ tuvieron una tasa de crecimiento superior a la de $6.0 / \mathrm{m}^{2}$ hasta el final de la fase experimental. El mayor incremen- to en peso se dio entre los 45 y 75 días del cultivo en ambas densidades de siembra.

En la Figura 6 se presenta la frecuencia relativa (\%) de la distribución de tallas ( $\mathrm{mm})$, tanto para hembras como para machos al inicio, cuando fue la misma población en el tanque de pre-cría para ambas densidades de siembra, y al final del cultivo. Puede observarse la distribución multimodal de machos compuesta por los individuos pequeños, medianos y grandes, así como la distribución unimodal de las hembras durante todo el periodo de estudio. Las hembras detuvieron su crecimiento a partir de los dos meses de cultivo, mientras que la población de los machos más grandes y de tenazas azules continuó aumentando, tanto en tamaño como en proporción de individuos, sobre todo en los sembrados a menor densidad.

La reducción del crecimiento de las hembras a partir del segundo mes de cultivo en ambas densidades de siembra estuvo relacionado a su fecundidad. Como se observa en la Figura 7, el porcentaje de hembras ovígeras se incrementó en el transcurso del cultivo sin existir mayores diferencias con respecto a la densidad de siembra. En la misma figura se aprecia que los machos de tenazas azules aparecieron a partir del primer mes de cultivo y su proporción llegó a ser considerablemente superior en la densidad de siembra más baja en el último mes.

La distribución por peso de los machos y las hembras en las dos densidades de siembra se muestra en la Figura 8. Se aprecia el crecimiento de tipo exponencial entre los 45 y 60 días (de 5 a $20 \mathrm{~g}$ de ganancia de peso) en ambas densidades de siembra. Asimismo, ambos sexos a partir de los 60 días alcanzaron una tasa de crecimiento superior en la densidad de siembra más baja, superando las hembras incluso a los machos de la mayor densidad. Como consecuencia, los pesos finales fueron significativamente superiores $(\mathrm{p}<0.01)$, tanto para las hembras $(27.4 \mathrm{~g})$ como para los machos (40.6 g) en la menor 


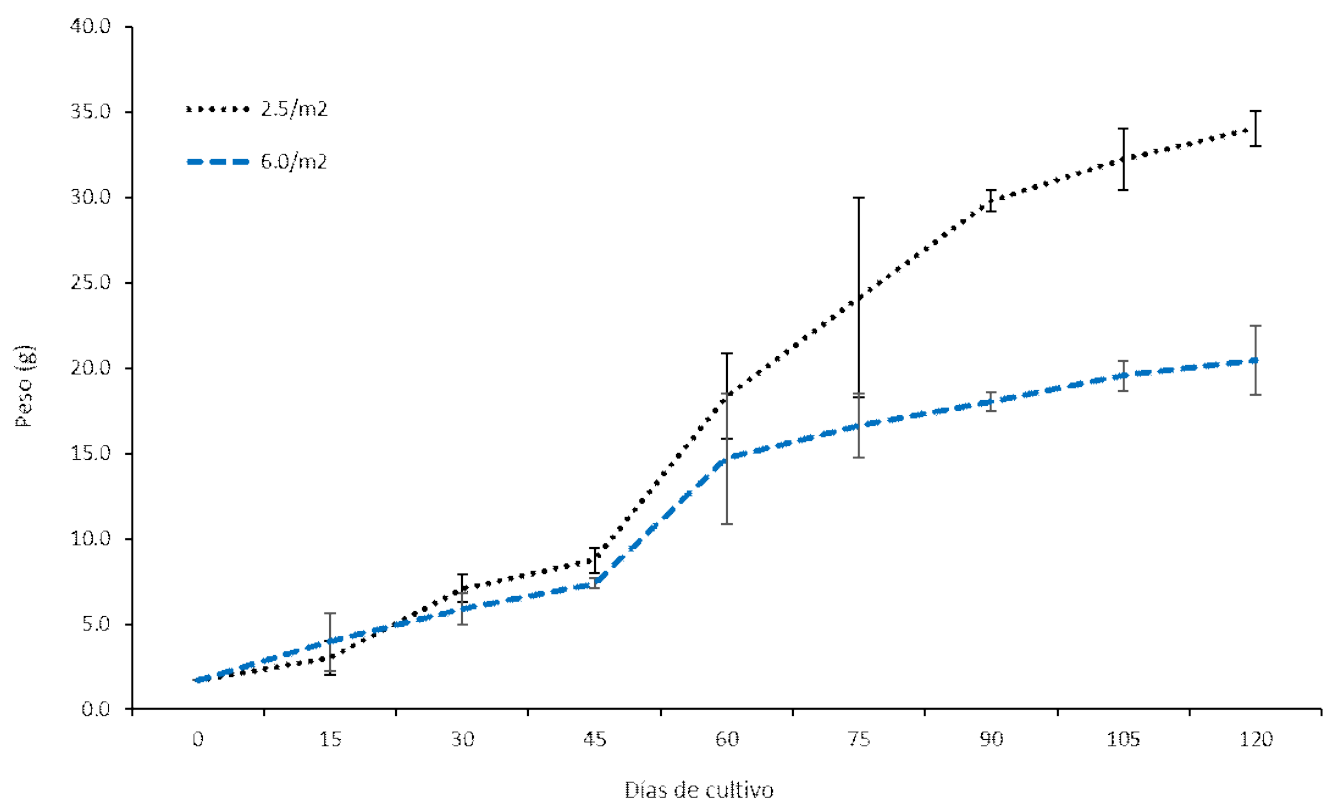

Figura 5. Incremento en peso de langostinos de agua dulce Macrobrachium rosenbergii bajo dos densidades de cultivo
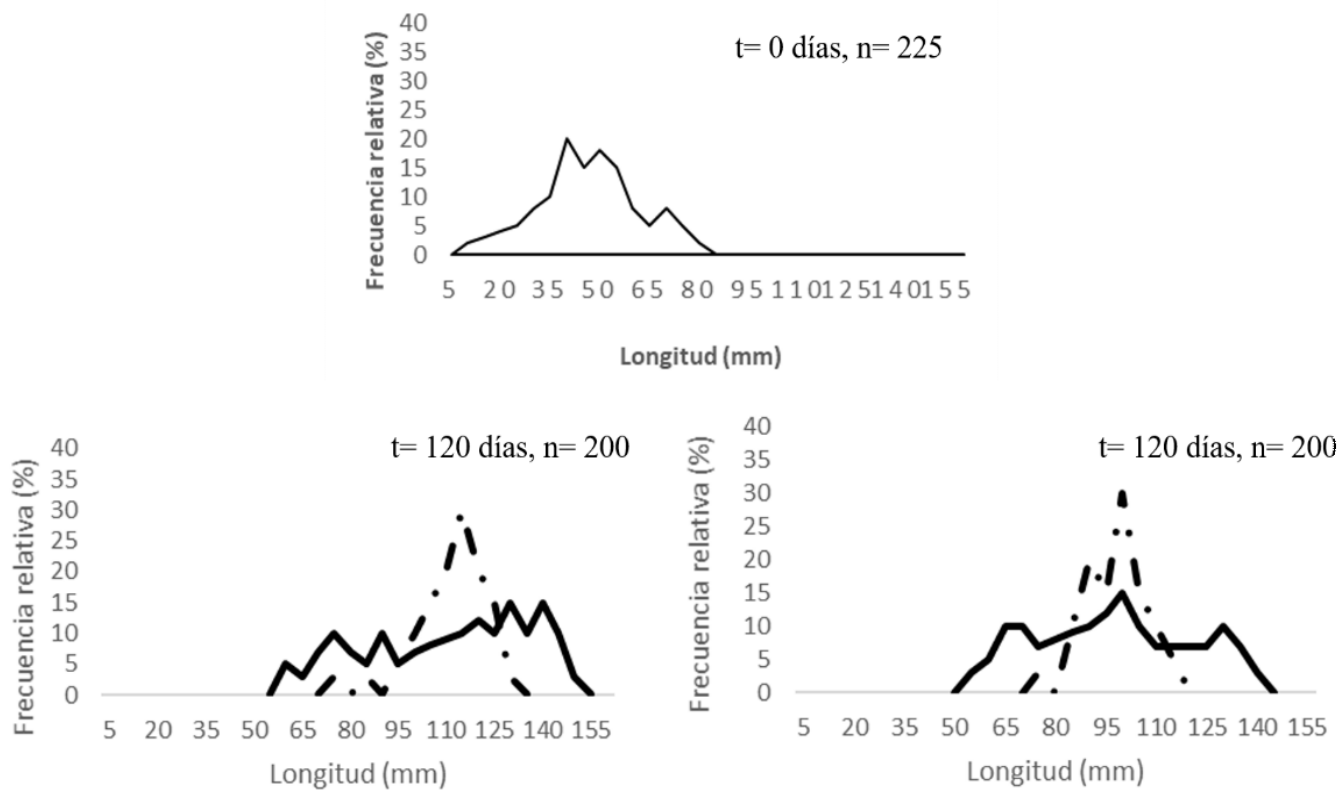

Figura 6. Distribución frecuencia relativa-longitud a 0-120 días mostrando la distribución multimodal de machos y unimodal de hembras de langostinos de agua dulce Macrobrachium rosenbergii bajo dos densidades de cultivo 


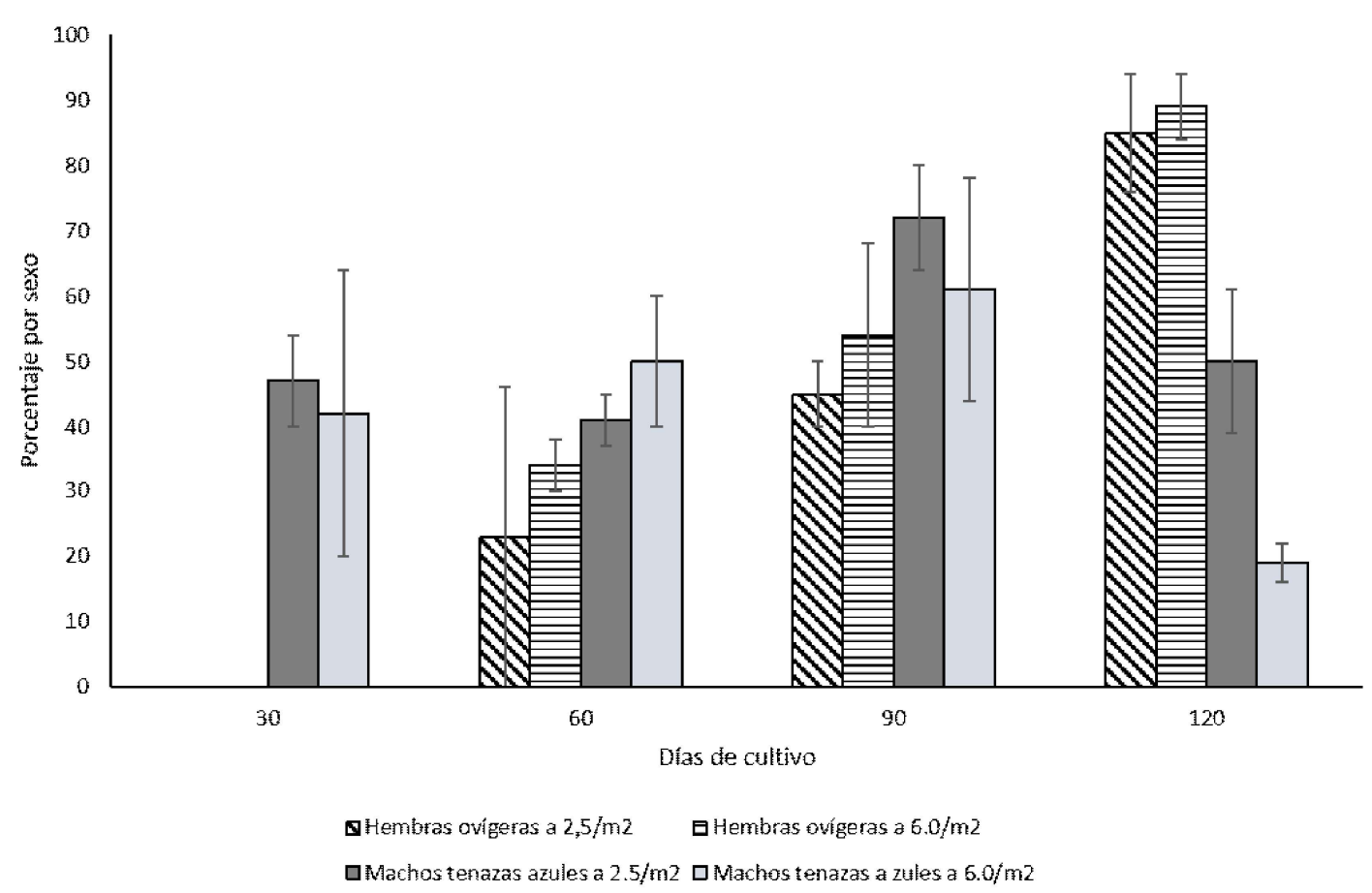

Figura 7. Porcentaje de hembras ovígeras y de machos de tenazas azules dentro de la población de langostinos de agua dulce Macrobrachium rosenbergii del mismo sexo en ambas densidades de siembra en el transcurso del cultivo

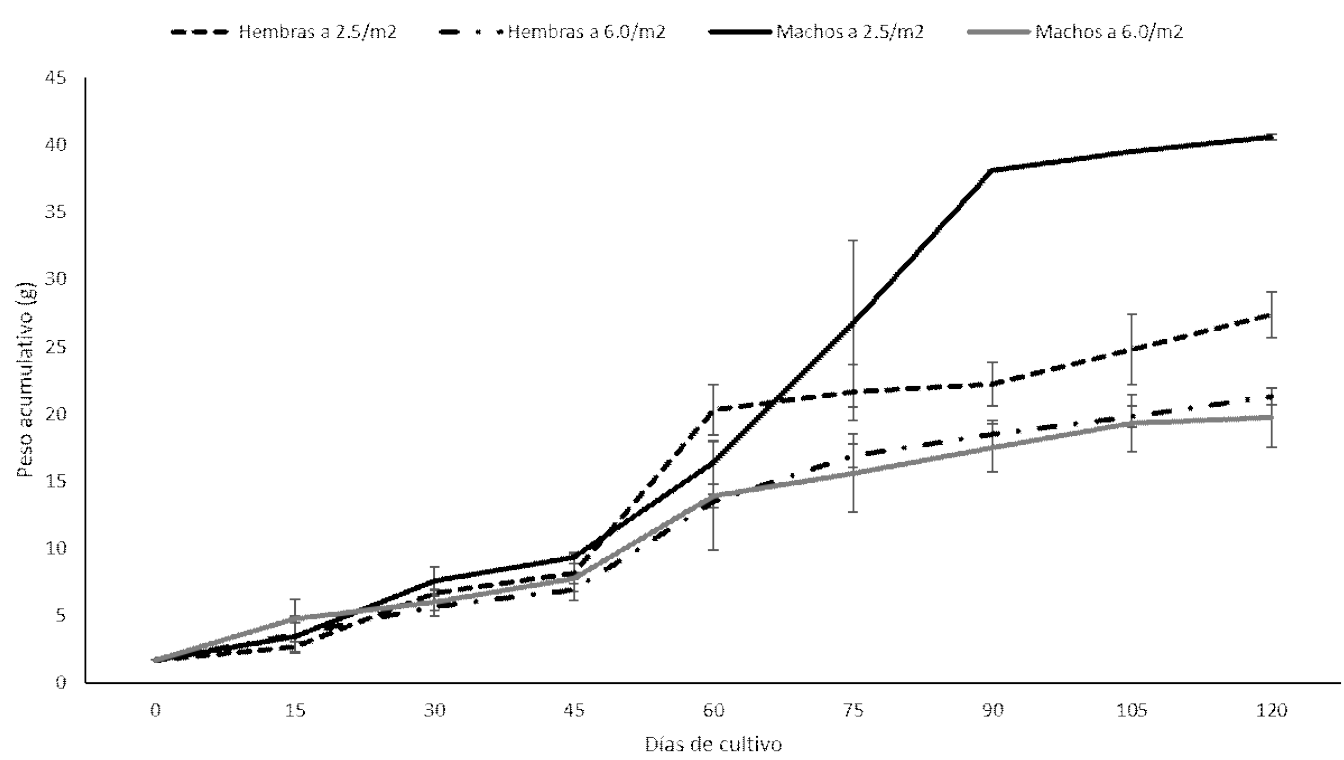

Figura 8. Curva de crecimiento y pesos finales de machos y hembras de langostinos de agua dulce Macrobrachium rosenbergii en dos densidades de siembra durante el periodo de cultivo 


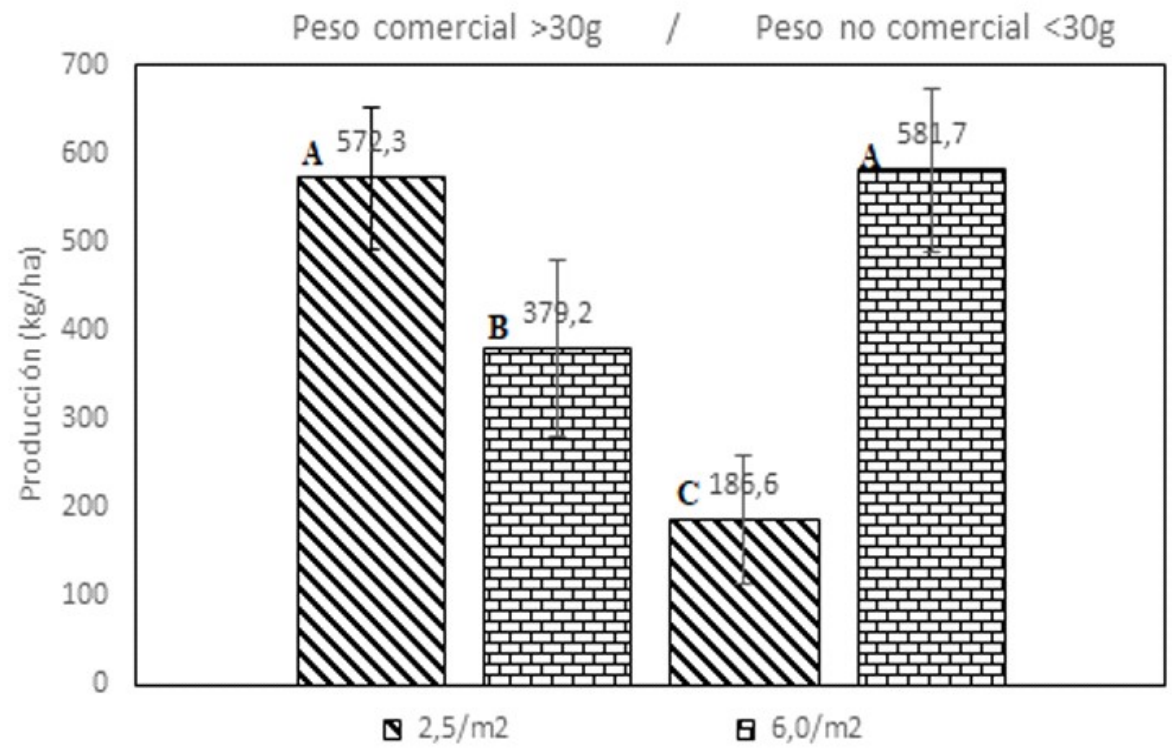

Figura 9. Producción comercial y no comercial de langostinos de agua dulce Macrobrachium rosenbergii en dos densidades de siembra. Variables con letras diferentes (A,B,C) indican diferencia significativa $(\mathrm{p}<0.01)$

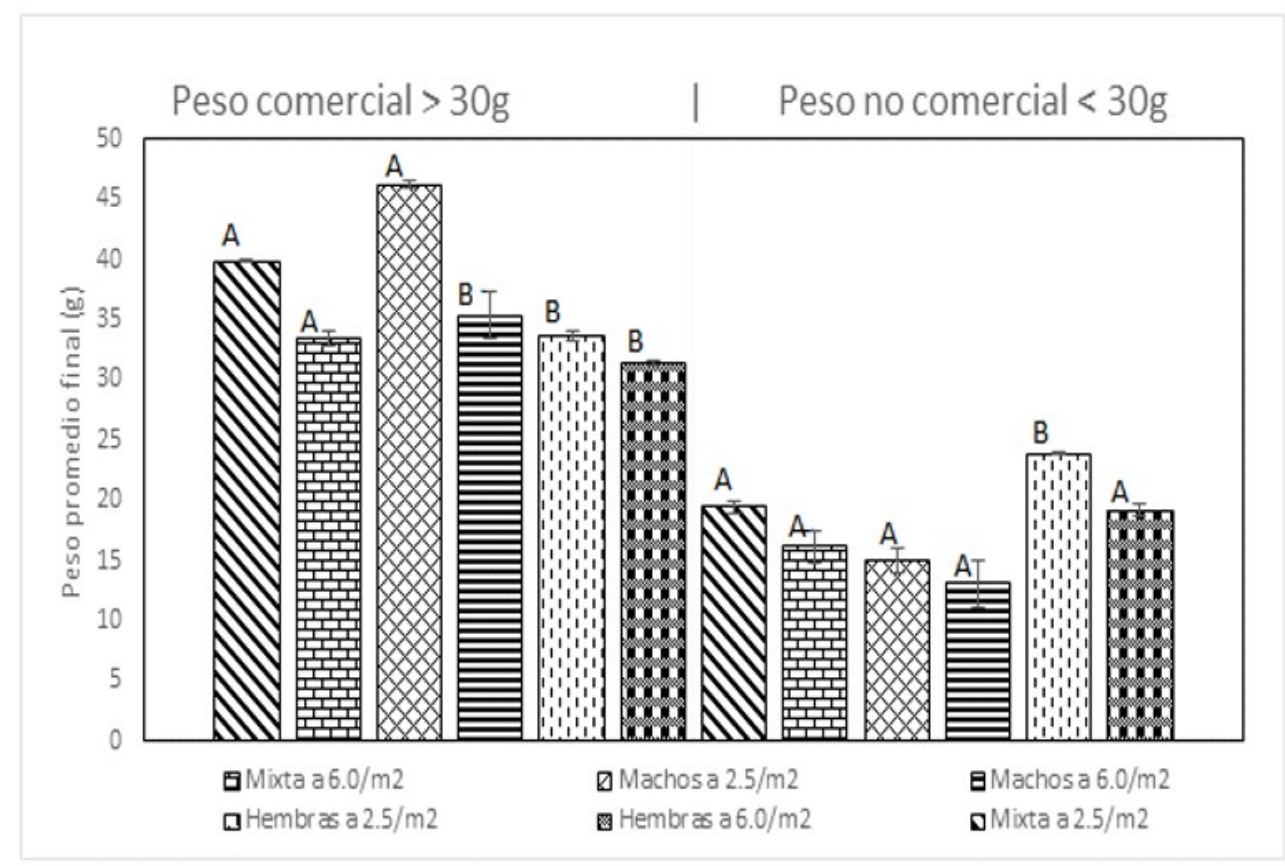

Figura 10. Peso promedio de la población mixta y por sexo con talla comercial y no comercial de langostinos de agua dulce Macrobrachium rosenbergii en dos densidades de siembra. Variables con letras diferentes (A, B) indican diferencia significativa $(\mathrm{p}<0.01)$ 


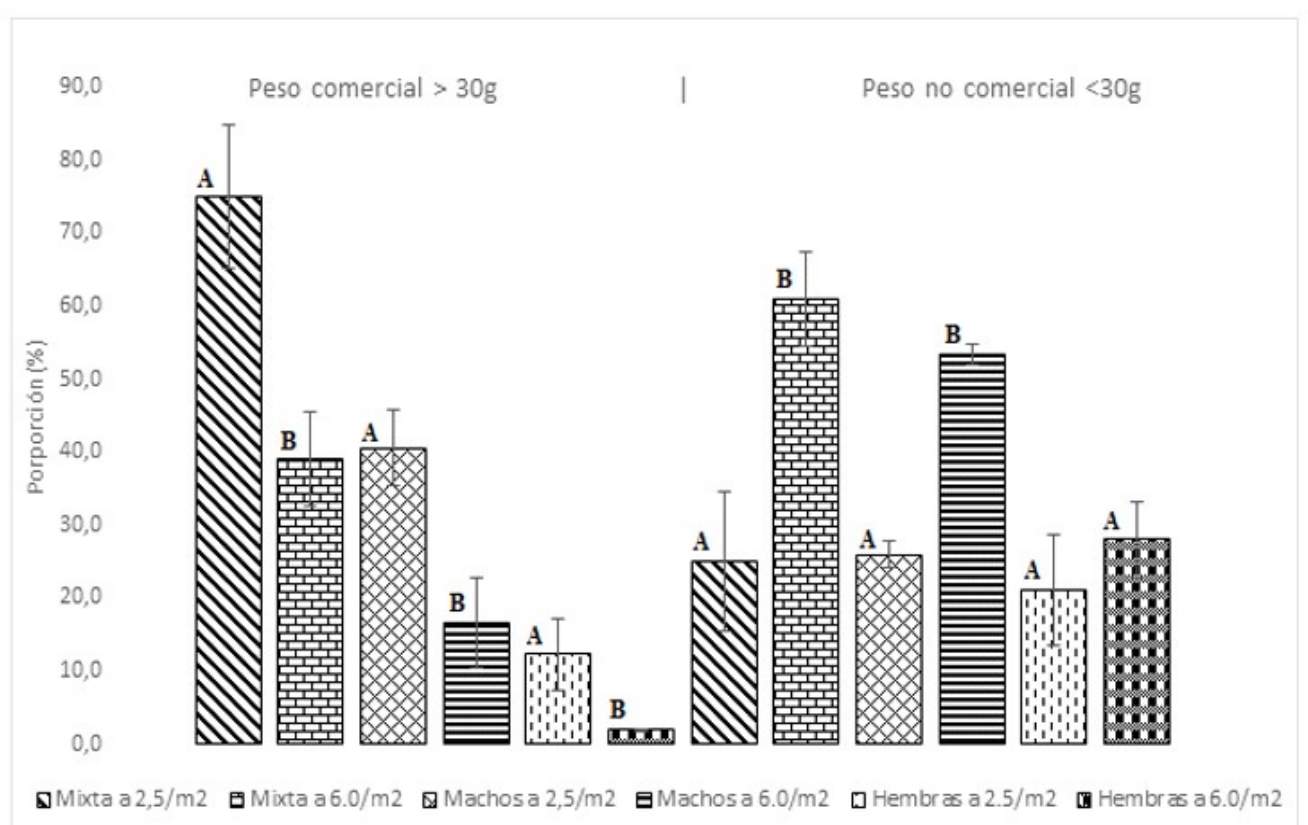

Figura 11. Proporción de la población mixta y por sexo con talla comercial y no comercial de langostinos de agua dulce Macrobrachium rosenbergii en dos densidades de siembra. Variables con letras diferentes $(A, B)$ indican diferencia significativa $(\mathrm{p}<0.01)$

Peso comercial $>30 \mathrm{~g}$ | Peso no comercial $<30 \mathrm{~g}$

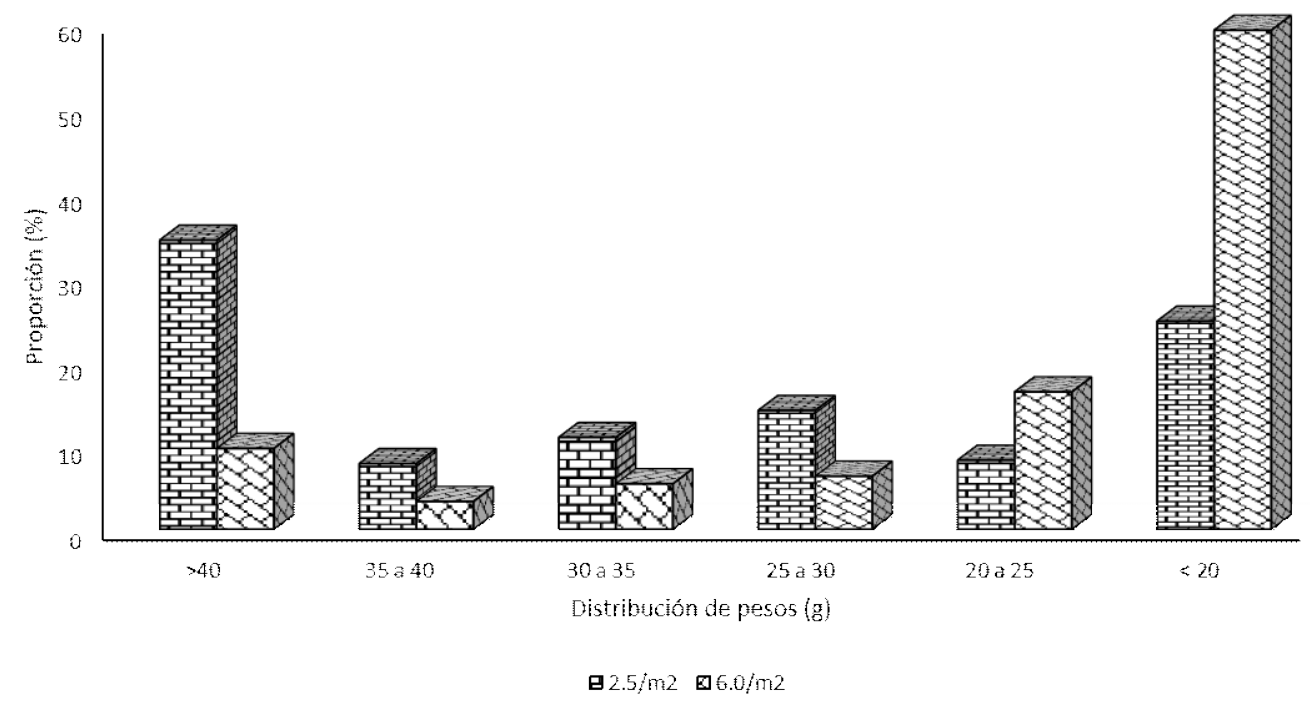

Figura 12. Proporción de langostinos Macrobrachium rosenbergii cosechados por intervalo de tallas comerciales y no comerciales. 


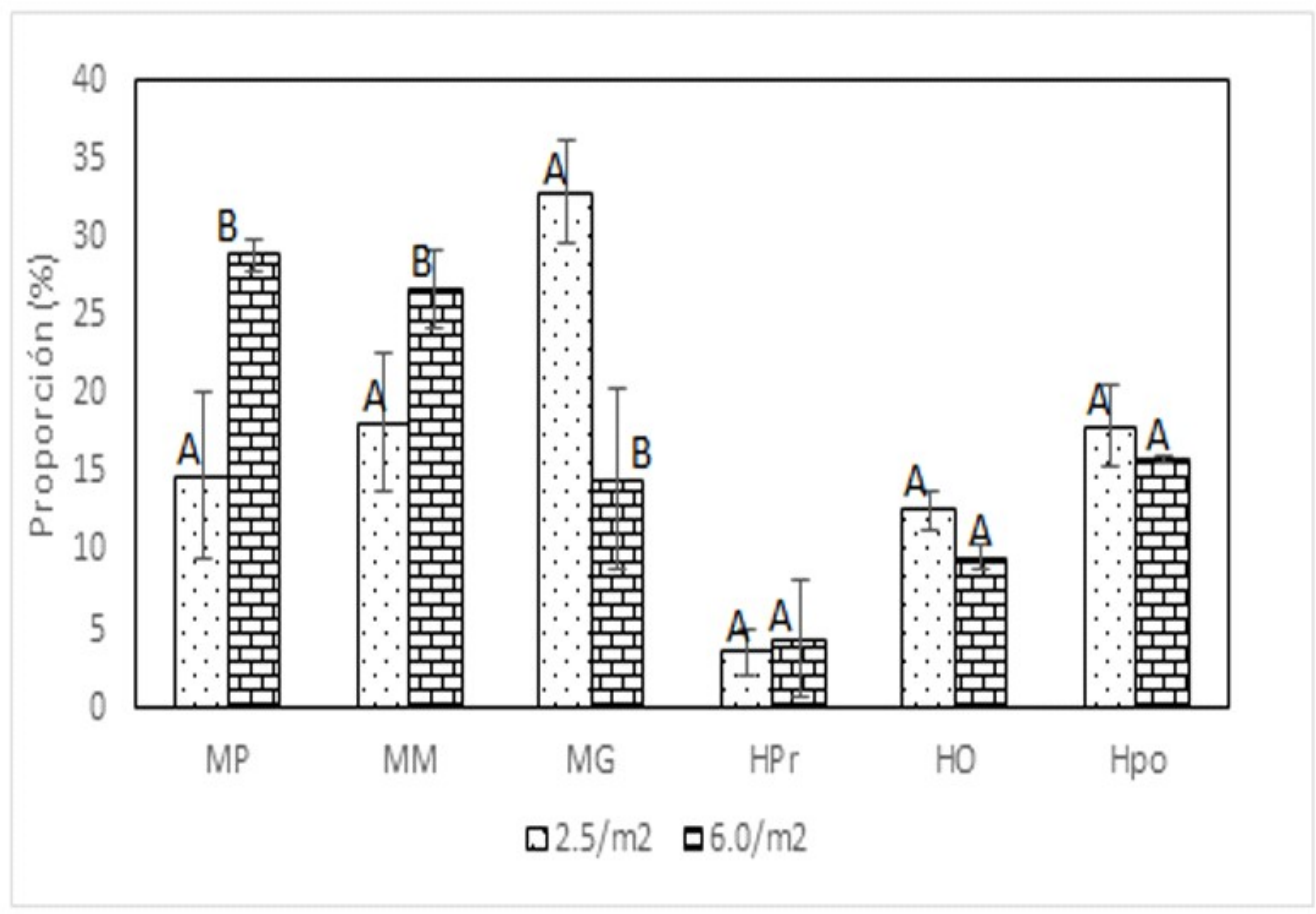

Figura 13. Proporción de langostinos Macrobrachium rosenbergii por morfotipo de machos y estado reproductivo de las hembras en estanques con dos densidades de siembra. $\mathrm{MP}=$ machos pequeños; $\mathrm{MM}=$ machos medianos; $\mathrm{MG}=$ machos grandes; $\mathrm{HPr}=$ hembras pre-ovígeras; $\mathrm{HO}=$ hembras ovígeras; $\mathrm{HPo}=$ hembras pos-ovígeras. Variables con letras diferentes $(A, B)$ indican diferencia significativa $(p<0.01)$.

densidad de siembra y de las hembras en baja densidad con respecto a los machos en alta densidad (19.7 g). La superioridad zootécnica de los machos en baja densidad sobre las hembras también fue significativa $(\mathrm{p}<0.01)$, mientras que los pesos fueron similares para ambos sexos en la densidad de siembra más alta.

Las características de la producción mostraron el dimorfismo sexual presente en la población de langostinos en ambas densidades de siembra. Estos se clasificaron en talla comercial con pesos superiores a los 30 $\mathrm{g}$ y en tallas no comercial con pesos inferiores. Puede observarse en la Figura 9 que la producción comercial es significativamente superior en la menor densidad de siembra con respecto a la más alta $(\mathrm{p}<0.01)$.
No hubo diferencias significativas entre los pesos promedios finales en ambos tipos de producciones entre tratamientos $(p<0.01)$, excepto por los machos en la producción comercial y las hembras en la producción no comercial, que tuvieron un peso significativamente superior en la menor densidad de siembra con respecto a los del mismo sexo en la densidad de siembra más alta $(\mathrm{p}<0.01)$ (Figura 10).

Las proporciones fueron superiores $(p<0.01)$ en la población mixta en menor densidad de siembra $(75 \%)$ con respecto a la más alta (39\%) en la producción comercial, mientras que el caso contrario (25 vs $61 \%$, respectivamente) ocurrió en la producción no comercial $(\mathrm{p}<0.01)$ (Figura 11). Igual fenó- 
meno ocurrió para la proporción de machos en ambos tipos de producciones, mientras que en las hembras solo se dio en la producción comercial. Un mayor porcentaje de machos que de hembras conformó la producción comercial en ambas densidades de siembra, mientras que en la no comercial solo los sembrados a $6.0 / \mathrm{m}^{2}$ mostraron esa superioridad con respecto a las hembras.

En la Figura 12 se presentan los porcentajes de langostinos cosechados con diferentes clases de pesos, diferenciándose entre los de talla comercial y no comercial. Se observa un mayor porcentaje (34.3\%) de los langostinos con tallas superiores a los $40 \mathrm{~g}$ en la densidad de siembra más baja con respecto a la más alta $(9.6 \%)$, mientras que el caso contrario ocurrió con pesos inferiores a los $20 \mathrm{~g}$ (24.7 y $59.2 \%$, respectivamente).

En la proporción por morfotipos de machos hubo diferencias significativas $(p<0.01)$ entre las densidades de siembra, pero las hembras clasificadas por su estado reproductivo como pre-ovígeras (HPr), ovígeras (HO) y pos-ovígeras (HPo) no mostraron ninguna afectación (Figura 13). Entre machos, la proporción de los pequeños (MP) (29\%) y medianos (MM) (27\%) en la densidad de siembra más alta fue significativamente superior que en la más baja ( 15 y $18 \%$, respectivamente), mientras que el caso contrario ocurrió con los machos grandes (MG) (33 y 15\%, respectivamente) $(\mathrm{p}<0.01)$. Asimismo, hubo el doble de machos pequeños a $6.0 / \mathrm{m}^{2}$ y de machos grandes a $2.5 / \mathrm{m}^{2}$ con respecto al mismo morfotipo en la otra densidad de siembra.

\section{Rentabilidad}

El precio por kilo de langostinos en Costa Rica se ha basado en la opinión de quienes tienen viveros y fincas con producto esporádico para el mercado. Actualmente en Costa Rica es de 6,248 colones por kilogramo los de talla no comercial $(<30 \mathrm{~g})$ y de 7,384 los de talla comercial ( $>30 \mathrm{~g})$. A un tipo de cambio actual de 568 colones por US\$, los precios anteriores corresponden a $\$ 11.0 / \mathrm{kg}$ y
Cuadro 1. Rango de pesos (g) y de precios por kilo (USD) sugeridos para langostinos $M$. rosenbergii frescos al pie de finca en 2019 en Costa Rica

\begin{tabular}{cccc}
\hline $\begin{array}{c}\text { Tamaño } \\
(\mathrm{g})\end{array}$ & US\$ & $\begin{array}{c}\text { Tamaño } \\
(\mathrm{g})\end{array}$ & US\$ \\
\hline $10-11$ & 5.00 & $22-24$ & 13.00 \\
$11-12$ & 5.75 & $24-25$ & 13.50 \\
$12-13$ & 6.50 & $25-26$ & 14.00 \\
$13-14$ & 7.25 & $26-27$ & 14.50 \\
$14-15$ & 8.00 & $27-28$ & 15.00 \\
$15-16$ & 8.75 & $28-29$ & 15.50 \\
$16-17$ & 9.50 & $29-30$ & 16.00 \\
$17-18$ & 10.25 & $30-32$ & 16.50 \\
$18-20$ & 11.00 & $32-36$ & 17.00 \\
$20-21$ & 11.50 & $36-40$ & 17.50 \\
$10-21$ & 12.00 & $40-45$ & 18.00 \\
$21-22$ & 12.50 & $46-50$ & 20.00 \\
\hline
\end{tabular}

$\$ 13.0 / \mathrm{kg}$ de manera respectiva. Según esos precios, el langostino pequeño de talla no comercial (18 g promedio) tiene un valor por unidad de peso de $0.62 \mathrm{USD} / \mathrm{g}$, mientras que los grandes de talla comercial ( $36 \mathrm{~g}$ promedio) tienen un valor por gramo inferior $(0.36$ USD/g). Los pesos promedios fueron obtenidos de ambas densidades de siembra en cada talla, Esto significa que el langostino a nivel nacional se cotiza mejor para los pequeños que para los grandes, lo cual no es congruente con el principio de que el precio es proporcional al tamaño de los langostinos. Por lo tanto, se hace necesario un ajuste en los precios de los langostinos a nivel nacional según las tallas como se muestra en el Cuadro 1 .

El resumen de los costos variables de producción en ambas densidades de siembra se presenta en el Cuadro 2. Del mismo se observa que los costos fueron más altos a $6.0 / \mathrm{m}^{2}(\$ 10,246)$ que a $2.5 / \mathrm{m}^{2}(\$ 4,796)$ con una diferencia de $\$ 5,450$. Esto fue debido 
Cuadro 2. Costos variables de producción para un ciclo de 120 días de langostinos Macrobrachium rosenbergii en un estanque de una hectárea

\begin{tabular}{|c|c|c|c|c|c|c|}
\hline $\begin{array}{l}\text { Densidad } \\
\text { de siembra }\end{array}$ & Rubro & Detalles & & $\begin{array}{l}\text { Costo } \\
\text { unidad } \\
\text { (USD) }\end{array}$ & $\begin{array}{c}\text { Costo/ha } \\
\text { (USD) }\end{array}$ & $\begin{array}{c}\text { Contribución } \\
(\%)\end{array}$ \\
\hline \multirow[t]{5}{*}{$2.5 / \mathrm{m}^{2}$} & Alimento & $1,214 \mathrm{~kg}$ & $\begin{array}{l}(48.6 \\
\text { sacos })\end{array}$ & $25 /$ saco & 1,215 & 26 \\
\hline & $\begin{array}{l}\text { Mano de } \\
\text { obra }\end{array}$ & $58.8 \mathrm{~h}$ & & $2.2 / \mathrm{h}$ & 130 & 3 \\
\hline & Biólogo & $0.5 \mathrm{~h}$ & $\begin{array}{l}(4 \\
\text { visitas })\end{array}$ & $49.3 / \mathrm{h}$ & 99 & 2 \\
\hline & Juveniles & 25,000 & & 0.13 & 3,250 & 69 \\
\hline & Total & & & & 4,694 & 100 \\
\hline \multirow[t]{5}{*}{$6.0 / \mathrm{m}^{2}$} & Alimento & $2,115 \mathrm{~kg}$ & $\begin{array}{l}(84.6 \\
\text { sacos })\end{array}$ & $25 /$ saco & 2,115 & 21 \\
\hline & $\begin{array}{l}\text { Mano de } \\
\text { obra }\end{array}$ & $58.8 \mathrm{~h}$ & & $2.2 / \mathrm{h}$ & 130 & 1 \\
\hline & Biólogo & $0.5 \mathrm{~h}$ & $\begin{array}{l}(4 \\
\text { visitas })\end{array}$ & $49.3 / \mathrm{h}$ & 99 & 1 \\
\hline & Juveniles & 60,000 & & 0.13 & 7,800 & 77 \\
\hline & Total & & & & 10,144 & 100 \\
\hline
\end{tabular}

sobre todo al incremento por la compra de mayor cantidad de semilla $(\$ 7,800$ vs $\$ 3,250)$ y de alimento $(\$ 2,115$ vs $\$ 1,215)$ en la densidad de siembra más alta. En efecto, la semilla pasó de representar el $69 \%$ del costo total en baja densidad de siembra al $77 \%$ en la densidad de siembra más alta. Luego sigue el alimento con un 21 y $26 \%$, respectivamente, mientras que el costo de la mano de obra no es significativo.

Considerando las conversiones alimenticias $\left(1.6: 1.0\right.$ a $2.5 / \mathrm{m}^{2}$ y $2.2: 1.0$ a $\left.6.0 / \mathrm{m}^{2}\right)$ y las producciones extrapoladas a 1 ha $(758.9$ y $961.2 \mathrm{~kg} / \mathrm{ha}$, respectivamente) en la fase experimental, se pudo obtener el gasto de alimento durante 120 días de cultivo en cada densidad de siembra, el cual fue de 1215 y $2115 \mathrm{~kg}$, respectivamente. El alimento es un pellet, con $30 \%$ de proteína, tamaño de 1.8 a $3.2 \mathrm{~mm}$ y viene en sacos de $25 \mathrm{~kg}$.

La mano de obra directa estuvo compuesta por una persona dedicada medio tiempo en las labores de pre-cría en tanques bajo techo y las de engorde en estanques de tierra donde se llevó a cabo la investigación. Se trata de un puesto de trabajo categorizado como de peón agrícola por el Ministerio de Trabajo de Costa Rica (MTSS, 2019) quien recibe el salario mínimo de ley. El desglose de las labores se presenta en el Cuadro 3 para un tipo de cultivo como el de esta investigación.

La mano de obra indirecta está compuesta por un profesional en biología que dedica al menos media hora mensual exclusivamente al proyecto, sin considerar su tiem- 
Cuadro 3. Desglose de la mano de obra directa en las labores del engorde de langostinos Macrobrachium rosenbergii en un estanque de una hectárea

\begin{tabular}{|c|c|c|c|c|}
\hline \multirow{2}{*}{ Labor } & \multirow{2}{*}{$\begin{array}{c}\text { Horas } \\
(\mathrm{n})\end{array}$} & \multicolumn{2}{|c|}{ Costo $^{1}$} & \multirow{2}{*}{ Características } \\
\hline & & (USD) & $\%$ & \\
\hline $\begin{array}{l}\text { Acondicionamiento } \\
\text { del estanque }\end{array}$ & 2 & 4.43 & 3 & Mantenimiento \\
\hline $\begin{array}{l}\text { Preparación del } \\
\text { estanque }\end{array}$ & 2 & 4.43 & 3 & Encalado \\
\hline Limpieza del estanque & 2 & 4.43 & 3 & Chapeas, misceláneos \\
\hline Siembra & 2 & 4.43 & 3 & $\begin{array}{l}\text { Colecta y traslado de } \\
\text { juveniles }\end{array}$ \\
\hline Alimentación & 24 & 53.14 & 41 & $0.2 \mathrm{~h} / \mathrm{d} \times 120$ días \\
\hline $\begin{array}{l}\text { Medición oxígeno y } \\
\text { temperatura }\end{array}$ & 3.4 & 7.53 & 6 & $0.2 \mathrm{~h} /$ semana $\mathrm{x} 17$ semanas \\
\hline $\begin{array}{l}\text { Muestreo de los } \\
\text { langostinos }\end{array}$ & 3.4 & 7.53 & 6 & $0.2 \mathrm{~h} /$ semana $\mathrm{x} 17$ semanas \\
\hline $\begin{array}{l}\text { Cosecha de los } \\
\text { langostinos }\end{array}$ & 8 & 17.71 & 14 & $\begin{array}{l}2 \text { personas por } 4 \mathrm{~h} \text { en } \\
\text { vaciado y recolecta }\end{array}$ \\
\hline Manejo post cosecha & 12 & 26.57 & 20 & $\begin{array}{l}3 \text { personas por } 4 \mathrm{~h} \text { en } \\
\text { selección por tamaños }\end{array}$ \\
\hline Total & 58.8 & 130.2 & 100 & \\
\hline
\end{tabular}

${ }^{1} 2.21 \mathrm{USD} / \mathrm{h}$

Cuadro 4. Costo de inversión y depreciación del estanque, materiales y equipo para el engorde de langostinos Macrobrachium rosenbergii en un estanque de una hectárea

\begin{tabular}{lcccc}
\hline \multirow{2}{*}{ Materiales y equipo } & \multicolumn{2}{c}{ Valor } & Vida útil & $\begin{array}{c}\text { Depreciación } \\
\text { USD/0.33 } \\
\text { años }\end{array}$ \\
\cline { 2 - 3 } & $\begin{array}{c}\text { USD/10 } \\
\text { has }\end{array}$ & USD/1 ha & (años) & $\begin{array}{c}\text { Und } \\
\text { 1 estanque }\end{array}$ \\
1 atarraya & 44.01 & 4.170 .00 & 10 & 390.00 \\
1 red & 264.08 & 26.41 & 5 & 0.29 \\
1 medidor oxígeno & 440.14 & 44.01 & 5 & 1.74 \\
1 tanque & 88.03 & 8.80 & 5 & 2.90 \\
Varias herramientas & 70.42 & 7.04 & 5 & 0.58 \\
1 balanza & 88.03 & 8.80 & 5 & 0.46 \\
3 baldes & 26.41 & 2.64 & 5 & 0.58 \\
\hline Total & $1,021.12$ & 1272.12 & & 0.17 \\
\hline
\end{tabular}


Cuadro 5. Resumen de los costos de producción por ciclo ( $\$ /$ ha por 4 meses), por unidad $(\$ / \mathrm{kg})$ y por año (\$/ha/año) de langostinos Macrobrachium rosenbergii en cada densidad de siembra

\begin{tabular}{llccc}
\hline $\begin{array}{l}\text { Densidad } \\
\text { de siembra }\end{array}$ & Tipo de costo & $\begin{array}{c}\text { Monto de } 4 \\
\text { meses } \\
(\$ / \mathrm{ha})\end{array}$ & $\begin{array}{c}\text { Costo } \\
\text { unitario } \\
(\$ / \mathrm{kg})\end{array}$ & $\begin{array}{c}\text { Costos anuales } \\
(\$ / \text { ha/año })\end{array}$ \\
\hline $2.5 / \mathrm{m}^{2}$ & Variables & $4,694.00$ & 6.19 & $12,673.80$ \\
& Fijos & $1,272.12$ & 1.68 & $3,434.40$ \\
& Depreciación & 396.70 & 0.52 & $1,071.10$ \\
\hline \multirow{2}{*}{$6.0 / \mathrm{m}^{2}$} & Total & $5,253.82$ & 8.39 & $17,179.30$ \\
& Variables & $10,144.00$ & 10.55 & 27388.80 \\
& Fijos & $1,272.12$ & 1.32 & $3,434.40$ \\
& Depreciación & 396.70 & 0.41 & $1,071.10$ \\
\hline & Total & $11,812.82$ & 12.28 & $31,894.3$ \\
\hline
\end{tabular}

Cuadro 6. Resumen de los ingresos por ciclo y por año de cultivo de langostinos Macrobrachium rosenbergii en cada densidad de siembra

\begin{tabular}{lrr}
\hline & $2.5 / \mathrm{m}^{2}$ & $6.0 / \mathrm{m}^{2}$ \\
\hline Producción total $(\mathrm{kg} / \mathrm{ha})$ & 756.40 & 960.90 \\
Producción comercial $(>30 \mathrm{~g})(\mathrm{kg} / \mathrm{ha})$ & 567.30 & 379.20 \\
Peso promedio $(\mathrm{g})$ & 39.90 & 33.40 \\
Proporción de la producción $(\%)$ & 75.00 & 39.00 \\
Precio promedio $(\$ / \mathrm{kg})$ & 13.00 & 13.00 \\
Sub total de ingresos $(\$ / \mathrm{ha})$ & $7,374.90$ & $4,929.60$ \\
\hline Producción no comercial $(<30 \mathrm{~g})(\mathrm{kg} / \mathrm{ha})$ & 189.10 & 581.70 \\
Peso promedio $(\mathrm{g})$ & 19.50 & 16.20 \\
Proporción de la producción $(\%)$ & 25.00 & 61.00 \\
Precio promedio $(\$ / \mathrm{kg})$ & 11.00 & 11.00 \\
Sub total de ingresos $(\$ / \mathrm{ha})$ & $2,080.10$ & $6,398.70$ \\
Precio ponderado $(\$ / \mathrm{kg})$ & 12.50 & 11.80 \\
\hline Total de ingresos $(\$ / \mathrm{ha})$ & $9,455.00$ & $11,328.30$ \\
\hline Ingresos anuales $(\$ / \mathrm{ha} / \mathrm{año})$ & $25,528.50$ & $30,586.40$ \\
\hline
\end{tabular}


Cuadro 7. Productividad, ingresos totales, costos variables, depreciación y costos fijos que determinaron la rentabilidad del engorde de langostinos Macrobrachium rosenbergii en dos densidades de siembra durante un año

\begin{tabular}{|c|c|c|c|c|c|c|}
\hline 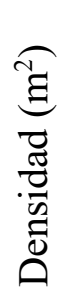 & 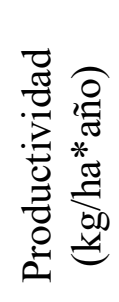 & 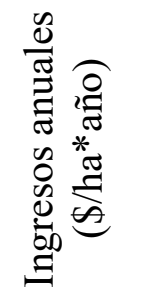 & 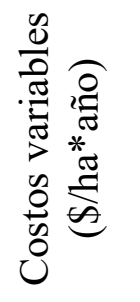 & 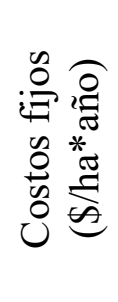 & 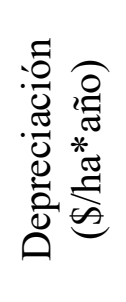 & 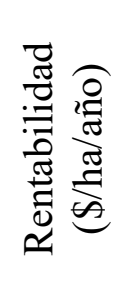 \\
\hline 2.5 & 2,042 & 25,528 & 12,674 & 3,434 & 1,071 & 8,349 \\
\hline 6.0 & 2,594 & 30,586 & 27,389 & 3,434 & 1,071 & $-1,308$ \\
\hline
\end{tabular}

Cuadro 8. Flujo de caja proyectado a cinco años en una crianza de langostinos Macrobrachium rosenbergii a una densidad de siembra de $2.5 / \mathrm{m}^{2}$

\begin{tabular}{lrrrrr}
\hline Vida económica (años) & 1 & 2 & \multicolumn{1}{c}{3} & \multicolumn{1}{c}{4} & \multicolumn{1}{c}{5} \\
\hline Ingresos(\$/ha/año) & 25,528 & 25,528 & 25,528 & 25,528 & 25,528 \\
Costos (\$/ha/año) & 12,674 & 12,674 & 12,674 & 12,674 & 12,674 \\
UAI $^{1}$ & 12,854 & 12,854 & 12,854 & 12,854 & 12,854 \\
UDI $^{2}$ & 8,998 & 8,998 & 8,998 & 8,998 & 8,998 \\
Inversión inicial & & & & & 4,505 \\
Capital de trabajo & & & & & 12,674 \\
Utilidad neta (\$/ha/año) & 8,998 & 8,998 & 8,998 & 8,998 & $-8,181$ \\
\hline
\end{tabular}

${ }^{1}$ Utilidad antes de impuestos

${ }^{2}$ Utilidad después de impuestos

Cuadro 9. Valor Actual Neto (VAN) del proyecto de una crianza de langostinos Macrobrachium rosenbergii a una densidad de siembra de $2.5 / \mathrm{m}^{2}$

\begin{tabular}{|c|c|c|c|c|c|c|c|}
\hline \multirow[t]{2}{*}{ Años } & \multirow[t]{2}{*}{ Ingresos } & \multirow[t]{2}{*}{ Costos } & \multirow[t]{2}{*}{ Utilidad } & \multirow[t]{2}{*}{$\begin{array}{c}\text { Tasa de } \\
\text { descuento } \\
(28 \%)\end{array}$} & \multicolumn{3}{|c|}{$\begin{array}{l}\text { Valor presente a una tasa de } \\
\text { descuento del } 28 \% \\
\text { (VP) }\end{array}$} \\
\hline & & & & & Ingresos & Costos & Utilidad \\
\hline 1 & 25,528 & 12,674 & 8,998 & 2.2442 & 57,290 & 28,443 & 20,193 \\
\hline 2 & 25,528 & 12,674 & 8,998 & 2.2442 & 57,290 & 28,443 & 20,193 \\
\hline 3 & 25,528 & 12,674 & 8,998 & 2.2442 & 57,290 & 28,443 & 20,193 \\
\hline 4 & 25,528 & 12,674 & 8,998 & 2.2442 & 57,290 & 28,443 & 20,193 \\
\hline 5 & 25,528 & 12,674 & $-8,181$ & 0.2910 & 7,429 & 3,688 & $-2,381$ \\
\hline VP & & & & & 236,588 & 117,460 & 78,393 \\
\hline
\end{tabular}


po de traslado hasta la unidad productiva ni su desplazamiento por la misma. Por lo general, en un horario de atención de 8 horas al día, un profesional puede atender al menos 20 ha de producción, lo que significa un tiempo de atención de 24 minutos por hectárea.

La semilla de langostino utilizada en precría fue la poslarva con un valor individual de $\$ 0.09$ (\$96.80/1000) obtenida en el vivero de Langostinos KoKo. Dado que en este estudio no fue posible establecer un costo real de cada juvenil, se acudió al estudio realizado por Valverde (1987), donde se estableció que el precio de la PL se incrementaba en $150 \%$ después de una etapa de pre-cría en estanques pequeños $\left(1000 \mathrm{~m}^{2}\right)$. Esta etapa tuvo una duración de 2 meses a una densidad de $50 / \mathrm{m}^{2}$, una sobrevivencia del $80 \%$ y una conversión alimenticia de 3.0:1.0. En el presente caso, el precio se incrementaría a $\$ 0.13$ por cada juvenil (\$132.00/1000) tomando como base el aumento descrito.

Dentro de los costos fijos de inversión se incluyen los dos estanques, así como los materiales y equipo utilizados en el estudio. Tomando en cuenta varios costos de construcción de estanques para langostinos, se llegó a obtener un promedio de $\$ 11,696 /$ ha. Este tipo de estanque tiene usualmente una vida útil corta, cercana a los 10 años, porque no poseen suficiente pendiente ni compactación de muros. Su valor actual es por lo tanto de $\$ 1,170$ y su depreciación durante los cuatro meses de cultivo $(\$ 1,170 \times 0.33)$ fue de $\$ 390$.

Los materiales y equipos utilizados con su respectivo valor, vida útil y depreciación se presentan en el Cuadro 4. Con los mismos se está en capacidad de atender una finca de al menos 10 ha por lo que se traslada el valor a 1 ha. El valor total fue de $\$ 1,272.12$ y la depreciación de $\$ 396.70$ para el estanque de 1 ha con los materiales y equipos incluidos.

El resumen de los costos de producción se presenta en el Cuadro 5 para las dos densidades de siembra. El costo unitario se obtuvo tomando en cuenta las producciones extrapoladas de 758.9 y $961.2 \mathrm{~kg} / \mathrm{ha}$ a $2.5 / \mathrm{m}^{2}$ y $6.0 / \mathrm{m}^{2}$, respectivamente, por ciclo con una duración de 120 días en el engorde de los langostinos. Si se asumen 15 días entre preparación de estanques, se pueden hacer 2.7 ciclos al año. Por lo tanto, la productividad es de 2049 y $2595 \mathrm{~kg} / \mathrm{ha} / \mathrm{año}$, respectivamente.

Los ingresos por concepto de ventas al pie de finca se presentan en el Cuadro 6. Al igual que en los costos de producción, se obtuvieron los ingresos anuales considerando 2.7 ciclos al año. El precio ponderado se obtuvo multiplicando la proporción de la cantidad cosechada en la producción comercial y no comercial con respecto al total por el precio en cada tipo de producción para ambas densidades de siembra. En la densidad de $2.5 / \mathrm{m}^{2}$ el precio ponderado fue de $\$ 12.5 / \mathrm{kg}$ y a $6.0 / \mathrm{m}^{2}$ fue de $\$ 11.8 / \mathrm{kg}$. Los costos unitarios fueron de $\$ 8.4 / \mathrm{kg}$ y $\$ 12.3 / \mathrm{kg}$, respectivamente. Por lo tanto, la ganancia fue de $\$ 4.1 / \mathrm{kg}$ a $2.5 / \mathrm{m}^{2}$ y no hubo ganancia, sino una pérdida, de $-\$ 0.5 / \mathrm{kg}$ a $6.0 / \mathrm{m}^{2}$. También se observa que a una densidad de siembra de $6.0 / \mathrm{m}^{2}$ se generaron más ingresos anuales $(\$ 11,328 / \mathrm{ha})$ por concepto de ventas que a $2.5 / \mathrm{m}^{2}(\$ 9,455 / \mathrm{ha})$, con una diferencia de US $\$ 1,873 /$ ha. Al ser más alta la productividad a $6.0 / \mathrm{m}^{2}(2595 \mathrm{~kg} / \mathrm{ha} / \mathrm{año})$ que a $2.5 / \mathrm{m}^{2}(2049 \mathrm{~kg} / \mathrm{ha} / \mathrm{año})$, las ganancias fueron mayores $(\$ 30,586$ vs $\$ 25,528)$ con una superioridad de $\$ 5,058 /$ ha/año. No obstante, en términos de utilidad por ciclo es más rentable la de $2.5 / \mathrm{m}^{2}$ porque con $758.9 \mathrm{~kg} /$ ha dio una utilidad de $\$ 4,201 /$ ha $(\$ 9,455$ - $\$ 5,254)$. Mientras tanto, a $6.0 / \mathrm{m}^{2}$ con una producción de $961.2 \mathrm{~kg} /$ ha se dieron pérdidas del orden de $-\$ 485 /$ ha $(\$ 11,328$ - $\$ 11,813)$ por ciclo de producción.

En el Cuadro 7 se presentan la productividad ( $\mathrm{kg} / \mathrm{ha} / \mathrm{año})$, los ingresos por concepto de ventas al pie de finca, los costos variables, la depreciación, el costo fijo y las utilidades brutas, sin considerar el pago del impuesto de ventas. Las utilidades brutas fueron de $\$ 8,349 / \mathrm{ha} /$ año en la densidad de siembra más baja mientras que no hubo rentabilidad $(-\$ 1,308)$ en la más alta. El punto de equilibrio para el valor del producto en la densi- 
dad de siembra más baja tomando en cuenta el costo variable por año fue en promedio de $\$ 6.18(\$ 12,674 / 2049 \mathrm{~kg})$ y en la más alta de $\$ 10.56(\$ 27,389 / 2594 \mathrm{~kg})$. Mientras tanto, el punto de equilibrio para el nivel de producción considerando el costo variable por año fue de $1014 \mathrm{~kg} / \mathrm{ha} /$ año $(\$ 12,674 / \$ 12.5)$ a $2.5 / \mathrm{m}^{2}$ y $2321 \mathrm{~kg} / \mathrm{ha} / \mathrm{año}(\$ 27,389 / \$ 11.8)$ a $6.0 / \mathrm{m}^{2}$.

Tomando en cuenta que la densidad de siembra de $2.5 / \mathrm{m}^{2}$ fue la rentable en este estudio, se le utilizó para el análisis financiero, tal y como se muestra a continuación:

- Utilidad bruta (ingresos anuales - costos totales anuales): $\$ 25,528-17,179=$ $\$ 8,349$

- Tasa Interna de Retorno (utilidad bruta/ inversión + costos de operación) $* 100$ : $\$ 8,349 /(4,505+12,674) * 100=48.6 \%$.

- Ganancia por kilo (precio $/ \mathrm{kg}$ - costo $/ \mathrm{kg}$ ) $\$ 12.5 / \mathrm{kg}-\$ 8.4 / \mathrm{kg}=\$ 4.10$.

- Periodo para pagar la inversión (inversión / utilidad bruta): $\$ 17,179 / 8,349=2.1$ años.

El flujo de caja del proyecto a cinco años en la densidad de siembra de $2.5 / \mathrm{m}^{2}$ se muestra en el Cuadro 8 y el Valor Actual Neto (VAN) en el Cuadro 9. Se incluye el capital de trabajo para cubrir los costos de producción del primer año. La tasa de descuento utilizada es del 28\%. Del Cuadro 9 se nota que el VAN es positivo $(\$ 78,393)$ con esta tasa de descuento y la relación beneficio costo es de US $\$ 2.0(236,588 / 117,460)$, lo que significa que por cada dólar invertido se ganan dos dólares.

\section{Discusión}

La temperatura ambiental reportada en este estudio se puede considerar alta provocando elevada evapotranspiración. No obstante, está dentro del rango recomendado $\left(>20{ }^{\circ} \mathrm{C}\right.$ por al menos 7 meses o un óptimo de $25-30{ }^{\circ} \mathrm{C}$ todo el año) y con poca varia- ción diurna o estacional (Muir y Lombardi, 2000) para la crianza de langostinos. La precipitación promedio mensual puede considerarse como muy baja ya que se encuentra cercana a los meses con la mínima precipitación (50-100 mm) medida en casi todas las estaciones de CORBANA en la región Caribe del país (CORBANA, 2013), debido al fenómeno de El Niño imperante.

El periodo de cultivo dependiendo de la variación de las lluvias es un factor crítico a la hora de seleccionar el sitio para la construcción de estanques (Muir y Lombardi, 2000). La mayor precipitación en estos meses también reduce al mínimo la radiación solar, con lo cual se evita el crecimiento de plantas acuáticas en el fondo de los estanques con poca profundidad operacional y del fitoplancton en la superficie del agua como en este caso.

En términos generales, la calidad del agua en los estanques abastecidos por aguas subterráneas durante el periodo de estudio no presentó problemas debido principalmente a que se obtenía con niveles adecuados, se utilizaron bajas densidades de siembra y se aplicaron pequeñas cantidades de alimento. La temperatura medida estuvo dentro del rango considerado aceptable $\left(24-34^{\circ} \mathrm{C}\right)$ y óptimo $\left(26-32^{\circ} \mathrm{C}\right)$ para langostinos en zonas tropicales (Boyd y Zimmermann, 2000). El valor promedio del oxígeno disuelto encontrado en ambos estanques también estuvo arriba del valor (>2 mg/l) recomendado al amanecer con un mínimo de $3 \mathrm{mg} / 1$ durante el día para no causar condiciones de estrés. Dependiendo de los lugares por donde pase, el agua subterránea podría traer niveles bajos de oxígeno disuelto y presencia de ciertos gases y metales disueltos como hierro, sulfuro de hidrógeno y metano, entre otros (Muir y Lombardi, 2000). La turbidez del agua estuvo dentro del rango $(40-50 \mathrm{~cm})$ recomendado para mantener niveles adecuados de oxígeno disuelto. Es un indicativo que no existió abundancia descontrolada del fitoplancton (sobre todo cianobacterias) que son comu- 
nes y bajan considerablemente las concentraciones de oxígeno disuelto en el agua (Nair y Salin, 2012).

El valor promedio de los demás parámetros químicos como $\mathrm{pH}$, nitratos, nitritos, nitrógeno amoniacal, amoníaco, fosfatos, alcalinidad, dureza total y por calcio en los dos estanques durante el periodo de estudio estuvo dentro de los rangos considerados ideales para el engorde de $M$. rosenbergii en estanques (Boyd y Zimmermann, 2000). Esto a pesar de que se utilizó agua proveniente del subsuelo en una época climatológica considera crítica. Hubo muy poco o ningún control en el manejo de los principales parámetros físicoquímicos como se hace comúnmente por medio de los recambios diarios de agua en fuentes superficiales de abastecimiento. El agua estancada sin recambios diarios tiene la ventaja de tener menos fluctuaciones provocadas por diferencias con el agua entrante.

Las densidades de siembra elegidas en el engorde están dentro del rango utilizado comúnmente con fines comerciales. En sistemas extensivos se incluye la de $2.5 / \mathrm{m}^{2}$ (1$4 / \mathrm{m}^{2}$ ) (Valenti y New, 2000) y en semi intensivos la de $6.0 / \mathrm{m}^{2}\left(4-20 / \mathrm{m}^{2}\right)$ (Valenti et al., 2010) en el monocultivo de langostinos en estanques de engorde. En EEUU se reportan siembras de $2.5 / \mathrm{m}^{2}$ (Tidwell, 2012), mientras que en Brasil se han reportado densidades de 5 a 10/m² (Marques y Moraes-Valenti, 2012).

El peso promedio de los juveniles sembrados estuvo dentro del rango $(0.2 \mathrm{a} 2.0 \mathrm{~g})$ utilizado en otros estudios. El aumento en el tamaño de siembra aumentó la producción en un $29 \%$ cuando se pasó de 0.2 a $0.7 \mathrm{~g}$ a una densidad de $4 / \mathrm{m}^{2}$. También, la población de los separados por tamaños aumentó el peso y la producción promedio finales en un $37 \mathrm{y}$ $46 \%$, respectivamente, después de cinco meses sembrados a $3.0 / \mathrm{m}^{2}$ (Tidwell y D'Abramo, 2000). Los pesos obtenidos a los cuatro meses en este estudio están dentro del rango logrado (20-30 g) al cabo de 4-6 meses en Brasil con densidades de siembra que han fluctuado entre 5 y 10/ $\mathrm{m}^{2}$ (Marques y Moraes-Valenti, 2012) y en regiones templadas $(20-50 \mathrm{~g})$ sembrando a $2.5 / \mathrm{m}^{2} \sin$ substratos artificiales (Tidwell, 2012). Diversos estudios desde 1975 hasta la fecha han mostrado la relación inversa que existe entre la densidad de siembra y el peso promedio individual a la cosecha tanto en policultivo como en monocultivo (Karplus y Sagi, 2010).

El crecimiento mostrado se ajusta al obtenido por medio de la ecuación de von Bertalanffy con un crecimiento tipo exponencial que empieza lento las primeras semanas, se incrementa en las siguientes y luego decae (Valenti y New, 2000). En este estudio se nota que los langostinos sembrados a $2.5 / \mathrm{m}^{2}$ tuvieron una tasa de crecimiento superior a la de $60 / \mathrm{m}^{2}$ hasta el final de la fase experimental. El mayor incremento en peso se dio entre los 45 y 75 días de cultivo en ambas densidades de siembra. Un patrón similar ha sido descrito con el aumento en la densidad de 4 a $20 / \mathrm{m}^{2}$, acentuándose de $4 \mathrm{a}$ $12 / \mathrm{m}^{2}$ y reduciéndose de 12 a $20 / \mathrm{m}^{2}$, lo que indica que, en bajas densidades de siembra, el efecto sobre el crecimiento de los langostinos es muy marcado, incluso en diferencias tan pequeñas como de 1 individuo $/ \mathrm{m}^{2}$ (Valenti y New, 2000).

Los valores reportados de sobrevivencia (80-90\%) fueron superiores al rango generalmente encontrado (50-80\%) y al considerado aceptable $(>50 \%)$ (Valenti y New, $2000)$. Está dentro de las más buenas $(>80 \%)$ (Marques y Moraes-Valenti, 2012). Al no detectarse organismos muertos en el transcurso del experimento, se presume que estos fueron depredados por los mismos langostinos, debido a su agresividad intraespecífica, sobre todo después de los periodos de muda cuando quedan suaves y más susceptibles a ser atacados.

La falta de significancia estadística en las conversiones alimenticias indica que este parámetro parece ser independiente de la densidad de siembra, además de que la ali- 
mentación suplementaria no se considera un factor crítico para el crecimiento de $M$. rosenbergii, pues los langostinos pueden obtener su dieta principalmente del medio natural. En países como Bangladés se han utilizado mayores cantidades de alimento con similares densidades de siembra obteniendo conversiones alimenticias más altas (2.3:1.0) (Ahmed, 2013), mientras que en India, las conversiones de alimento han sido similares (1.8-2.0:1.0) en poblaciones mixtas (Nair y Salin, 2012). Las tasas de alimentación estuvieron dentro del rango $\left(12-28 \mathrm{~g} / \mathrm{m}^{2} / \mathrm{d}\right)$ reportado en zonas templadas (EEUU) para langostinos sembrados entre 2.0 y $2.5 / \mathrm{m}^{2}$ (Tidwell, 2012).

Las producciones reportadas en este estudio son similares a las establecidas por Marques y Moraes-Valenti (2012) en Brasil. Con densidades de siembra de 5 a $10 / \mathrm{m}^{2}$, lograron una producción mínima de $300 \mathrm{~kg} / \mathrm{ha}$, un promedio de $800-1500 \mathrm{~kg} /$ ha y un máximo de $2500 \mathrm{~kg} / \mathrm{ha}$. Con la línea genética proveniente del sudeste asiático (Myanmar), Tidwell et al (2014) obtuvieron, al cabo de 112 días, una producción de $475 \mathrm{~kg} / \mathrm{ha}$ con una densidad de siembra de $2.5 / \mathrm{m}^{2}$. En zonas templadas con ciclos de producción de 120 a 140 días sembrando juveniles de 0.3 a $0.5 \mathrm{~g}$ provenientes de pre-cría a una densidad promedio de $2.5 / \mathrm{m}^{2}$, se ha obtenido después de 4.0 4.5 meses, $1000 \mathrm{~kg} / \mathrm{ha}$ con un peso final de 20-30 g (Tidwell y D'Abramo, 2010).

La productividad obtenida en este estudio fue de $2049 \mathrm{~kg} / \mathrm{ha} /$ año a $2.5 / \mathrm{m}^{2}$ y 2595 $\mathrm{kg} / \mathrm{ha} / \mathrm{año} \mathrm{a} 6.0 / \mathrm{m}^{2}$. Ambas fueron obtenidas con una estrategia de producción extensiva y un bajo nivel de tecnología. En zonas tropicales con similares condiciones de clima, las productividades han fluctuado entre $1000 \mathrm{y}$ $3000 \mathrm{~kg} / \mathrm{ha} / \mathrm{año}$ con sistemas semi-intensivo de cultivo en estanque abastecidos por agua superficial (Valenti y New, 2000). Asimismo, Valenti et al., (2010) reportan producciones de 1500 a $4500 \mathrm{~kg} / \mathrm{ha} / \mathrm{año}$ en zonas subtropicales al igual que Tidwell (2012) en EEUU sembrando a $2.5 / \mathrm{m}^{2}$ con 2-3 ciclos al año con pre-crías.
La distribución multimodal de machos compuesta por los pequeños, medianos y grandes y unimodal de las hembras durante todo el periodo de estudio es la común en la población de langostinos cultivados en estanques (Karplus et al., 2000). En los machos se da un tipo de conducta agonística por territorio y alimento, pero sobre todo por reproducción (Karplus y Sagi, 2010). El macho de alargadas tenazas azules fecunda la hembra y la protege por 2-3 días hasta que esta endurece el exoesqueleto, mientras que los de tenazas anaranjadas pueden fecundarlas, pero no la protegen por su conducta sexual incompleta. La habilidad de los machos enanos les permite fecundar la hembra receptiva pasando entre ella y el macho de tenazas azules que la protege.

Los datos confirmaron la relación inversa existente entre el peso promedio final, tanto para hembras como machos, y la densidad de siembra. La disminución del peso de los machos en la mayor densidad de siembra se debió sobre todo a la gran heterogeneidad de tallas en esta población, lo cual ha sido demostrado en muchos estudios dentro de un amplio rango de densidades de siembra (Karplus y Sagi, 2010). La mayor proporción de machos pequeños conforme aumenta la densidad de siembra ha estado relacionada a su mayor interacción con los machos grandes que les limitan su crecimiento. Este cambio en la proporción de machos ya ha sido descrito en densidades de $1-4 / \mathrm{m}^{2}$, mientras que en densidades de $0.5-1.5 / \mathrm{m}^{2}$ se consideró invariable con proporciones de 5:4:1 entre machos pequeños, medianos y grandes (Karplus et al., 2000).

Los precios establecidos en este estudio dan un amplio rango de valores dependiendo del tamaño del producto. Es útil establecer un precio estándar según un rango de peso de los langostinos, como se hace en camarones marinos donde el precio aumenta en 0.18 USD por cada gramo de incremento en peso a partir de los $10 \mathrm{~g}$ de peso corporal (Valverde-Moya y Alfaro-Montoya, 2014). Los que existían eran muy subjetivos entre 
langostinos pequeños o grandes, le daban más valor al primero que al segundo por unidad de peso y solo consideraban 2 o 3 precios. En Brasil se clasifican por tamaños que van de 15 a $25 \mathrm{~g}$ (pequeños), 25 a $35 \mathrm{~g}$ (medianos) y 35 a $45 \mathrm{~g}$ (grandes) y los precios han fluctuado entre $\$ 5$ a $\$ 18 / \mathrm{kg}$ con un incremento aproximado de $\$ 1.0$ de un tamaño al otro (Marques y Moraes-Valenti, 2012). Estos especímenes se vendían en Langostinos KoKo entre $\$ 10$ y $\$ 14 / \mathrm{kg}$ frescos con cabeza al pie de finca (New y Kutty, 2010). Estos precios están cercanos al promedio establecido a nivel internacional de $\$ 12 / \mathrm{kg}$ por Malecha (2012) dentro de un rango de $\$ 11.0$ a $\$ 13.2 / \mathrm{kg}$. En términos generales se estima que el precio se ha incrementado 17 veces en 35 años desde 1984 (New y Nair, 2012). De mantenerse el mismo nivel de incremento, el establecido por Valverde (1987) para los comerciables $(>30 \mathrm{~g}$ ) y los no comerciables $(<30 \mathrm{~g})$ serían en la actualidad de $\$ 12 / \mathrm{kg}$ y $\$ 9 / \mathrm{kg}$, respectivamente.

En términos generales, el costo de los estanques para langostinos se ha establecido en $\$ 11,132 /$ ha los de 4 ha y en $\$ 19,151 /$ ha los de 0.8 ha, sin entrada por gravedad de agua fresca, en fincas ubicadas en Latinoamérica y el Caribe (Rhodes, 2000). En camarones marinos, los estanques ubicados en las cercanías de zonas de manglar en Costa Rica tienen un costo estimado en $\$ 17,606 /$ ha (Valverde-Moya y Alfaro-Montoya, 2014). Los estanques utilizados en este estudio de $300 \mathrm{~m}^{2}$ tuvieron un costo de construcción por concepto de excavación calculado en $\$ 1,408$. Valverde (1987) estimaba que un estanque de 1 ha se podía construir en 62 horas con un costo actualizado de $\$ 70 / \mathrm{h}(\$ 4,340 / \mathrm{ha}) \mathrm{du}-$ rante la época más seca de la región caribe costarricense. En el año 2000, se estimaba la inversión por hectárea en $\$ 6,253$ para un estanque debidamente construido con sus respectivos accesorios (Rhodes et al., 2010). Tomando en cuenta todos estos costos se puede obtener un promedio de $\$ 11,696 /$ ha.
Los ingresos por concepto de ventas al pie de finca fueron más altos a $6.0 / \mathrm{m}^{2}$ que a $2.5 / \mathrm{m}^{2}$ con una diferencia de US $\$ 1,873 /$ ha. Al ser más alta la productividad en esa densidad de siembra, también generó las mayores ganancias con una superioridad de $\$ 5,058 /$ ha/año. Esta superioridad fue debida sobre todo a la mayor producción por unidad de área a pesar de que el precio del producto fue inferior por tener un peso final más bajo.

No obstante, las ganancias por $\mathrm{kg}$ de producto fueron de $\$ 4.1$ a $2.5 / \mathrm{m}^{2}$, mientras que a $6.0 / \mathrm{m}^{2}$ se dieron pérdidas económicas de $-\$ 0.5 / \mathrm{kg}$. En Brasil se vendían a $\$ 5 / \mathrm{kg}$ los que costaban $\$ 3 / \mathrm{kg}$ por lo que el margen de utilidad era de $\$ 2 / \mathrm{kg}$ en 1990 (Rhodes et al., 2010). El valor por kilogramo de producto en el punto de equilibrio fue de $\$ 6.18$ en la densidad de siembra más baja y de $\$ 10.56$ en la más alta como promedio ponderado. En Kentucky, EEUU, se alcanzó el punto de equilibrio a $\$ 9 / \mathrm{kg}$ en alta densidad y de $\$ 17 / \mathrm{kg}$ en densidades bajas sembrando juveniles a un costo de \$80/1000 (Rhodes et al., 2010). El mismo ha bajado de $\$ 18.4 / \mathrm{kg}$ en 1991 a $\$ 9.93 / \mathrm{kg}$ en 2000 en zonas templadas, debido al mejoramiento en las tecnologías de cultivo. Para el cultivo tradicional con densidades de $4.0 / \mathrm{m}^{2}$ y sin substrato se estableció en $\$ 6.75 / \mathrm{kg}$ en 2004, similar al que se obtuvo en este estudio a $2.5 / \mathrm{m}^{2}$ (Tidwell y D'Abramo, 2010).

El nivel de producción en equilibrio fue de $1014 \mathrm{~kg} / \mathrm{ha} / \mathrm{año} \mathrm{a} 2.5 / \mathrm{m}^{2}$ y de $2321 \mathrm{~kg} / \mathrm{ha} /$ año a $6.0 / \mathrm{m}^{2}$. El mejoramiento en las producciones con densidades de siembra más altas lo puede alcanzar el productor instalando en su finca tres fases de cultivo como son la pre-cría $\left(75 / \mathrm{m}^{2}\right)$, el desarrollo $\left(10 / \mathrm{m}^{2}\right)$ y el engorde $\left(6 / \mathrm{m}^{2}\right)$ como hacían algunos productores de camarones en el Golfo de Nicoya en 2014 (Valverde-Moya y Alfaro-Montoya, 2014). Otra posibilidad es incrementar la producción de langostino pequeño si existe suficiente mercado, intensificando e implementando los cultivos continuos por medio del aumento en las densidades de siembra a 10 langostinos $/ \mathrm{m}^{2}$ hasta talla comercial, cosechas selectivas por 
tallas y la resiembra para substituir los que se van cosechando. Otra alternativa para mejorar la producción es el cultivo en monosexual de $\mathrm{Pl}$ 's $100 \%$ hembras, que se pueden sembrar en altas densidades (hasta $20 / \mathrm{m}^{2}$ ) presentando una tasa de crecimiento más uniforme que los machos, obviándose el crecimiento heterogéneo individual y las cosechas selectivas. Esta posibilidad tecnológica ya existe (Levi et al., 2017) con ventajas comerciales demostradas; sin embargo, se requiere su evaluación económica adaptada al país y las condiciones donde se va a implementar su cultivo.

La densidad de siembra rentable en el estudio fue la de $2.5 / \mathrm{m}^{2}$, porque con una producción de $758.9 \mathrm{~kg} / \mathrm{ha}$ dio una utilidad neta de $\$ 4,201 / \mathrm{ha}$. A 6.0/ $\mathrm{m}^{2}$ hubo pérdidas económicas de $-\$ 485 /$ ha. En Brasil se obtuvo la máxima utilidad ( $\$ 912 / \mathrm{ha}$ ) con una producción de $403 \mathrm{~kg} / \mathrm{ha}$, sembrando a $1.5 / \mathrm{m}^{2}$, después de ocho meses de cultivo (Rhodes et al., 2010). Según los mismos autores, cuando se pasó de 4.0 a $5.0 / \mathrm{m}^{2}$, la utilidad disminuyó de $\$ 3,277 /$ ha a $\$ 831 /$ ha. En este estudio, cuando se incrementó la densidad de siembra no solo se redujo la proporción de langostinos con talla comercial, sino que también se incrementaron considerablemente los costos de producción debido, sobre todo, al aumento en el costo de la semilla. Por lo tanto, la rentabilidad depende de la densidad de siembra en cultivos semi-intensivos con poca aplicación de tecnología como ocurrió en estos casos (Rhodes et al., 2010).

Las utilidades brutas también fueron positivas en la densidad de siembra más baja mientras que no hubo rentabilidad en la más alta. En Brasil se obtuvo una rentabilidad de $\$ 9,047 /$ ha/año sembrando a $4 \mathrm{Pl} / \mathrm{m}^{2}$ sin incluir los costos fijos y no hubo rentabilidad cuando las densidades de siembra se incrementaron de 12 a $20 / \mathrm{m}^{2}$. Las pérdidas económicas a $6.0 / \mathrm{m}^{2}$ se debieron sobre todo al incremento en más del doble de los costos variables de producción comandados por el costo de la semilla, conforme lo hizo la densidad de siembra. Estos resultados indican que las Pl's con ese precio no permiten incrementar las densidades de siembra, por lo que la demanda se vuelve inconsistente y sumamente baja para los propios viveros de producción de larvas. El productor de langostinos debe, por lo tanto, reducir los costos de producción, ya sea mejorando las producciones (mayor sobrevivencia y talla de cosecha), sembrando menos o incrementando el precio de venta ( $>\$ 20 / \mathrm{kg}$ como ocurre en Hawaii) (Rhodes et al., 2010).

El estudio de sensibilidad económica para la densidad de siembra rentable en este estudio $\left(2.5 / \mathrm{m}^{2}\right)$ indicó una TIR de $48.6 \% \mathrm{y}$ un periodo de 2.1 años para pagar la inversión. En Brasil, una TIR del 20\% se ha considerado muy atractiva en el monocultivo de poblaciones mixtas, con 4 años para pagar la inversión en fincas pequeñas de 1-2 ha (Almeida y Moraes-Valenti, 2012). En República Dominicana con una producción superior $(2800 \mathrm{~kg} / \mathrm{ha} / \mathrm{año})$ a la reportada en este estudio (2042 kg/ha/año) se logró pagar la inversión en 5 años. Esto para sistemas semiintensivos aplicando escasa tecnología de cultivo.

Un VAN positivo con esta tasa de descuento del $28 \%$ y la relación beneficio costo 2.0 indican que el negocio es factible económicamente a una densidad de siembra de $2.5 / \mathrm{m}^{2}$. Esta relación es superior a la obtenida por Valverde (1987) (1.62) en la misma densidad de siembra. En los cultivos semiintensivos, extensivos mejorados y extensivos en Bangladés se obtuvo un recobro de $\$ 1.75,1.74$ y 1.62 por cada $\$ 1.00$ invertido, respectivamente (Ahmed, 2013).

\section{Conclusiones}

- El manejo con estanques abastecidos con aguas subterráneas puede ser implementado por parte de acuicultores artesanales que no tienen aguas superficiales o donde existe el riesgo de estar contaminadas. 
- La siembra de juveniles provenientes de pre-cría tiene varias ventajas en este tipo de cultivos como el mejor control del sistema, dado que se pueden contar los que se siembran, se pueden reponer las posibles pérdidas por mortalidad en el engorde, son fáciles de mantener las condiciones óptimas del cultivo, se siembran juveniles más grandes y resistentes capaces de competir mejor por alimento y evitar los depredadores en el estanque, y se interrumpe la continuación del crecimiento diferencial que contribuye a la disparidad de tallas en el transcurso del cultivo.

- El precio a nivel de finca puede considerarse barato si no se le da un valor agregado ni se le busca un nicho de mercado que pague un valor considerablemente más alto para un producto de lujo tipo gourmet que ya preparado se considera una delicatés. Un platillo con 5 langostinos de 50 g puede ofrecerse a US\$20, por lo que de un kilo se haría US\$80.

- Según el análisis financiero, el productor nacional de langostinos debe ser capaz de tomar decisiones y hacer ajustes en el sistema de cultivo para adaptarlo a sus posibilidades económicas haciéndolo más eficiente. La estrategia de producción debe ser flexible para ajustarse al cambio, sobre todo del precio del producto en el mercado.

\section{Literatura Citada}

1. Ahmed, N. (2013). On-farm feed management practices for giant river prawn (Macrobrachium rosenbergii) farming in southwest Bangladesh. In: On-farm feeding and feed management in aquaculture 583: 269-301.

2. Almeida HL, Moraes-Valenti PM. 2012. Current status and prospects of farming the giant river prawn Macrobrachium rosenbergii (De Man, 1879) and the amazon river prawn Macro- brachium amazonicum (Heller, 1862) in Brasil. Aquac Res 984-992. doi: 10.1111/ j.1365-2109.2011.03032.x

3. Bhujel R. 2008. Statistics for aquaculture. Iowa, USA: Wiley-Blackwell. $240 \mathrm{p}$.

4. Boyd C, Zimmermann S. 2000. Growout systems: water quality and soil management. In: New WM, Valenti W (eds). Freshwater prawn culture: the farming of Macrobrachium rosenbergii. London: Blackwell-Science. $\mathrm{p}$ 221-238.

5. [CORBANA] Corporación Bananera Nacional. 2013. Clima, variabilidad y cambio climático en la Vertiente Caribe de Costa Rica: Un estudio básico para la actividad bananera. San José: Universidad de Costa Rica. 67 p.

6. Karplus I, Sagi A. 2010. The biology and management of size variation. In: New M, Valenti W, Tidwell J, D'Abramo L, Kuttty M (eds). Freshwater prawn biology and farming. Oxford: WileyBlackwell.p 316-345.

7. Karplus I, Malecha S, Sagi A. 2000. The biology and management of size variation. In: New MB, Valenti W (eds). Freshwater prawn culture: the farming of Macrobrachium rosenbergii. London: Blackwell Science. p 259-282.

8. Lazur A. 2007. Growout pond and water quality management. JIFSAN good aquacultural practices manual. USA: University of Maryland. USA. 17 p.

9. Levi T, Rosen O, Eliam B, Azulay D, Zohar I, Aflalo ED, Benet A, et al. 2017. All-female monosex culture in the freshwaer prawn Macrobrachium rosenbergii - A comparative large-scale field study. Aquaculture 479: 857-862.

10. Malecha S. 2012. The case for allfemales freshwater prawn, Macrobrachium rosenbergii (De Man), culture. Aquac Res 43: 1038-1048. doi: 10.1111/j.1365-2109.2011.03007.x

11. Marques H, Moraes-Valenti P. 2012. Current status and prospects of farming the giant river prawn (Macrobrachium 
rosenbergii De Man 1879) and the Amazon river prawn Macrobrachium amzonicum (Heller 1862) in Brasil. Aquac Res, 984-992.

12. [MTSS] Ministerio de Trabajo y Seguridad Social. 2019. Lista de salarios mínimos por ocupación año 2019. [Internet]. Disponible en: http://www.mtss.go.cr/temas-laborales/salarios/listasalarios.html\#

13. Muir J, Lombardi J. 2000. Grow-out systems - Site selection and pond construction. In: New M, Valenti W (eds). Freshwater prawn culture: the farming of Macrobrachium rosenbergii. London: Blackwell Science. p 126-156.

14. Nair M, Salin K. 2012. Current status and prospects of farming the giant river prawn Macrobrachium rosenbergii (De Man) and the monsoon river prawn Macrobrachium malcolmsonii. Aquac Res 43: 999-1014. doi: 10.1111/j.13652109.2011.03074.x

15. Na-Nakorn U. 2017. News/Reviving Giant Prawn Farming in Asia. In: Conference on Giant Freshwater Prawns. Thailand.

16. Na-Nakorn U, Jintasataporn O. 2012. Current status \& prospects of farming the giant-river prawn (Macrobrachium rosenbergii de Man 1879) in Thailand. Aquac Res 43: 1015-1022. doi: 10.1111/j.1365-2109.2011.03037.x

17. New M. 2000. History and global status of freshwater prawn farming. In New M, Valenti W (eds). Freshwater prawn culture: the farming of Macrobrachium rosenbergii. London: Blackwell Science. p 1-11.

18. New M. 2010. History and global status of freshwater prawn farming. In New M, Valenti W, Tidwell J, D'Abramo L, Kutty M (eds). Freshwater prawns biology and farming. Oxford: WileyBlackwell. p 1-11.

19. New M, Kutty M. 2010. Commercial freshwater prawn farming and enhancement around the world. In: New M, Valenti W, Tidwell J, D'Abramo L,
Kutty M (eds). Freshwater prawn biologya and farming. Oxfrod: Wiley-Blackwell. p 346-399.

20. New MB, Nair M. 2012. Global scale of freshwater prawn farming. Aquac Res 43: 960-969. doi: 10.1111/j.13652109.2011.03008.x

21. Peña N, Chacón J. 2019. Acuicultura en Costa Rica. Pesca y Medio Ambiente. [Internet]. Disponible en: https:// pescaymedioambiente.com/2019/07/03/ acuicultura-en-costa-rica-nelsonpen $\%$ CC\%83a-navarro-y-jonathanchacon-guzman/

22. Rhodes R. 2000. Economics and business management. In: New M, Valenti W (eds). Freshwater prawn culture: the farming of Macrobrachium rosenbergii. London: Blackwell Science. $\mathrm{p}$ 369-392.

23. Rhodes R, Hanson T, Dasgupta S. 2010. Economics and business management. In: New M, Valenti W, Tidwell J, D'Abramo L, Kutty M (eds). Freshwater prawn biology and farming. Oxford: Wiley-Blackwell. p 448-474.

24. Tidwell JH. 2012. Current status and prospects of farming the giant river prawn (Macrobrachoum rosenberggi De Man 1879) in the United States. Aquac Res 43: 1023-1028. doi: 10.1111/ j.1365-2109.2012.03130.x

25. Tidwell J, D'Abramo L. 2010. Growout systems - culture in temperate zones. In: New M, Valenti W, Tidwell J, D'Abramo L, Kutty M (eds). Freshwater prawn biology and farming. Oxford: Wiley-Blackwell. p 180-194.

26. Tidwell J, D'Abramo L. 2000. Growout sistems - Culture in temperate zones. In: New M, Valenti W (eds). Freshwater prawn culture: the farming of Macrobrachium rosenbergii. London: Blackwell Science. p 177-186.

27. Tidwell JH, Odeyemi OO, Coyle SD, Schneider KJ, Bright LA. 2014. Production comparison of three genetic strains of freshwaterprawn, Macrobrachium rosenbergii, raised under two pond management technologies. J World 
Aquac Soc 45: 595-603. doi: doi.org/ 10.1111/jwas. 12148

28. Valenti W, New M. 2000. Grow-out systems - monoculture. In: New M, Valenti W (eds). Freshwater prawn culture: the farming of Macrobrachium rosenbergii. London: Blackwell Science. p 157-176.

29. Valenti W, New M, Salin K, Ye J. 2010. Grow-out systems - monoculture. In: New M, Valenti W, Tidwell J, D'Abramo L, Kutty M (eds). Freshwater prawn biology and farming. Oxford: WileyBlackwell.p 154-179.

30. Valverde J. 1987. Efecto de la densidad de siembra en el crecimiento, sobrevivencia y características de la produc- ción de Macrobrachium rosenbergii (Decapoda:Palamonidae) monocultivado en estanques en 28 Millas, Limón, Costa Rica. Tresis de Grado. Heredia: Universidad Nacional. $144 \mathrm{p}$.

31. Valverde-Moya J, Alfaro-Montoya J. 2014. Productividad y rentabilidad del cultivo de camarones marinos en el Golfo de Nicoya, Costa Rica. Revista Ciencias Marinas y Costera 6: 37-53.

32. Varela-Mejías A, Valverde-Moya $J$. 2018. Determinación de la causa de mortalidad en un vivero del langostino gigante de agua dulce Macrobrachium rosenbergii en Costa Rica: análisis de caso. Rev Inv Vet Perú 29: 666-675. doi: 10.15381/rivep.v20i2.14522 